\title{
Time-Periodic Weak Solutions to Incompressible Generalized Newtonian Fluids
}

\author{
Anna Abbatiello \\ Communicated by E. Feireisl
}

\begin{abstract}
In this study we are interested in the Navier-Stokes-like system for generalized viscous fluids whose viscosity has a power-structure with exponent $q$. We develop an existence theory of time-periodic three-dimensional flows.
\end{abstract}

Mathematics Subject Classification. 35Q35, 76D05, 35Q30.

Keywords. Time-periodic solution, Weak solution, Global existence, Generalized Newtonian fluid, Viscous fluid.

\section{Introduction}

The Navier-Stokes-like system for an incompressible generalized viscous fluid reads

$$
\begin{aligned}
& \operatorname{div}_{x} \mathbf{v}=0 \text { in }(0, T) \times \Omega, \\
& \partial_{t} \mathbf{v}+\operatorname{div}_{x}(\mathbf{v} \otimes \mathbf{v})-\operatorname{div}_{x} \mathbb{S}+\nabla_{x} p=\mathbf{b} \text { in }(0, T) \times \Omega,
\end{aligned}
$$

where the viscous stress tensor $\mathbb{S}$ is a nonlinear function depending on the symmetric part of the velocity gradient $\mathbb{D} \mathbf{v}:=\frac{1}{2}\left(\nabla_{x} \mathbf{v}+\left(\nabla_{x} \mathbf{v}\right)^{t}\right)$, and particularly we are concerned with the following rheological law:

$$
\mathbb{S}=2 \mu_{0}\left(\kappa+|\mathbb{D} \mathbf{v}|^{2}\right)^{\frac{q-2}{2}} \mathbb{D} \mathbf{v} \text { with } \mu_{0}>0, \kappa \geq 0, q \geq 1,
$$

where for brevity we set $2 \mu_{0}=1$. The Navier-Stokes system is the outcome by setting $q=2$ or equivalently by setting the generalized viscosity equal to a constant. The existence and the regularity theories to the Navier-Stokes-like system (1.1) give rise to the discussion on the bounds for the exponent or power-law index $q$. The best result for the existence of weak solutions to the initial and boundary value problem in dimension $d$ requires $q>\frac{2 d}{d+2}$ and it is obtained by employing the Lipschitz truncations method, see [13]. Although the weak formulation is meaningful for any $q>1$, when $q<\frac{2 d}{d+2}$ the lack of compactness in the space $L^{2}(\Omega)$ may arise measures, particularly in the convective term $\mathbf{v} \otimes \mathbf{v}$, moreover below this critical exponent the concept of weak solution has some deficiencies. Indeed, there is a recent result [8] investigating the concept of weak solutions for low $q$ 's and showing that multiple Leray-Hopf solutions may emanate from the same $L^{2}$-initial-datum when $1<q<\frac{2 d}{d+2}$. However, any bound can be removed in the class of generalized dissipative solutions. They are introduced in [1] to a system describing the motion of a generalized viscous fluid and it is proved their long-time and large-data existence; particularly for a rheological law of type (1.1c) their existence holds for any $q \geq 1$. Despite the concept of generalized dissipative solution is weaker than the one of weak solution, it is showed in [1] that these solutions enjoy the weak-strong uniqueness principle, in other words a generalized dissipative solution must coincide with a classical solution as soon as and as long as the latter exists. Note that such notion of generalized dissipative solution is different from the concept of measure-valued solutions. Regarding the smoothness of the solutions to (1.1), there are only partial answers. Especially the existence of smooth solutions corresponding to smooth data in the three-dimensional case is open, see e.g. [21, Section 4] for a survey 
of regularity results. The uniqueness of weak solutions in the energy class holds for any $q \geq 1+\frac{2 d}{d+2}$, see $[20]$.

In this paper we are concerned with the existence of periodic in time solutions to system (1.1b)-(1.1c) in a domain $\Omega \subset R^{3}$, completed with the boundary condition

$$
\mathbf{v}_{\mid \partial \Omega}=0
$$

More specifically, we assume that the force datum is a periodic in time function and we show that there exists a weak solution to system (1.1) which exhibits a reproductive property in time and has the same period of the force datum. We are interested in the degenerate case of the rheological law (1.1c) indeed we achieve the result with

$$
\kappa \geq 0
$$

Concerning the nonlinear viscosity problem, this question was first partially answered by Lions [19] where it is assumed $q>3$ and $\mathbb{S}$ depends on the full gradient. In [9] the author considers the power-law exponent $q=q(x)$ a space-variable function but the convective term is neglected. In [3] the difficulties are overcome proving the existence of regular solutions under the assumption of smallness of the data, then it is established the existence of periodic in time solutions in a space-periodic domain with $q \in\left[\frac{5}{3}, 2\right)$. Further relevant results are [5] where the authors requires $q \in\left(\frac{9}{5}, 2\right)$ for the space-periodic case and [17] where the Womersley flow is analyzed. For other examples of time-periodic flows in the linear viscosity case see e.g. $[16,18,22,23]$. So far all the mentioned results concern time-periodic incompressible flows, however similar problems for compressible models have been addressed in e.g. [4,14,15].

In this paper we prove the existence of time-periodic weak solutions for any $q$ in the same range of values for which it is valid the existence of weak solutions to the initial and boundary value problem, i.e. $q>6 / 5$, see Theorem 1.3 below. Also, the problem is here analyzed in the three-dimensional case for simplicity but the result may be achieved in any spatial dimension $d$; then the supercritical case corresponds to $q \in\left(\frac{2 d}{d+2}, 1+\frac{2 d}{d+2}\right)$ and the subcritical/critical case to $q \geq 1+\frac{2 d}{d+2}$.

We construct time-periodic approximations following the idea in [24] used for the Navier-Stokes system. To this aim we add a laplacian term to Eq. (1.1b), then we perform a fixed point argument at the level of the Galerkin approximations and finally we take the limit in the approximations. The limit enjoys the reproductive property in time and is a weak solution to the system. We split the analysis into the two cases $q \geq 11 / 5$ and $q \in(6 / 5,11 / 5)$. Once the result is achieved for $q \geq 11 / 5$, then we can construct time-periodic approximations in the case $q \in(6 / 5,11 / 5)$ by adding a $p$-Laplacian term with $p=11 / 5$ to the system. (Note that a similar approximation scheme is exhibited in [6].) However, the case $q \in(6 / 5,11 / 5)$ is more delicate and requires the introduction of one more approximation level in the generalized viscosity, in order to ensure the uniqueness of the solution to the Galerkin system which is needed for the fixed point argument. Finally we take the limit first in the Galerkin approximation, then in the parameter $\kappa$ in the generalized viscosity (achieving in this way the result also for the degenerate case $\kappa=0$ ), and finally we get rid of the regularizing terms through a trick which only use that we can keep a power of the parameter $\varepsilon$ in the estimates uniform in $\varepsilon$, as e.g. in [6].

In the end the identification of the limit for the viscous stress tensor may be achieved through the standard Minty's trick when the limit of the velocity field itself can be used as test function in the weak formulation of $(1.1 \mathrm{~b})$, which is no longer possible when $q \in(6 / 5,11 / 5)$. In this case we need to perform the Lipschitz truncations technique and more specifically the problem is solved employing the solenoidal version developed in [7] which simplifies this technique significantly.

Finally, inspired by the results in [10] we analyze the property of extinction in a finite time for $q \in(6 / 5,2)$ and we prove that to the periodic extinction of the external force $\mathbf{b}$ corresponds a periodic extinction of the solution, see Corollary 1.5 below. 


\subsection{Time-Periodic Weak Solution and Main Result}

Definition 1.1. A field $\mathbf{f}:(0,+\infty) \times \Omega \rightarrow R^{3}$ is said to be time-periodic with period $T>0$ if

(i) $\mathbf{f} \in C([0, T] ; X)$ with $\left(X,\|\cdot\|_{X}\right)$ a Banach space;

(ii) $\mathbf{f}(t+T, \cdot)=\mathbf{f}(t, \cdot)$ in $X$ for any $t \geq 0$.

Definition 1.2. Assume $T>0, \kappa \geq 0, q>6 / 5$ and let $\mathbf{b} \in C\left([0, T] ; L^{2}\left(\Omega ; R^{3}\right)\right)$ be a time-periodic function with period $T$. We say that $\mathbf{v}$ is a time-periodic weak solution to system (1.1) with period $T$ if the following hold:

(i) $\mathbf{v} \in L^{\infty}\left((0, T) ; L^{2}\left(\Omega ; R^{3}\right)\right) \cap L^{q}\left((0, T) ; W_{0, \operatorname{div}}^{1, q}\left(\Omega ; R^{3}\right)\right), \partial_{t} \mathbf{v} \in\left(L^{q}\left((0, T) ; W_{0, \operatorname{div}}^{1, q}\left(\Omega ; R^{3}\right)\right)\right)^{*}$ if $q \geq 11 / 5$, or $\partial_{t} \mathbf{v} \in\left(L^{\frac{5 q}{5 q-6}}\left((0, T) ; W_{0, \operatorname{div}}^{1, \frac{5 q}{5 q-6}}\left(\Omega ; R^{3}\right)\right)\right)^{*}$ if $q \in(6 / 5,11 / 5)$;

(ii) $\mathbf{v} \in C\left([0, T] ; L^{2}\left(\Omega ; R^{3}\right)\right)$ if $q \geq 11 / 5$, or $\mathbf{v} \in C_{\text {weak }}\left([0, T] ; L^{2}\left(\Omega ; R^{3}\right)\right)$ if $q \in\left(\frac{6}{5}, \frac{11}{5}\right)$;

(iii) there exists $\mathbb{S} \in L^{q^{\prime}}\left((0, T) \times \Omega ; R^{3 \times 3}\right)$ such that

$$
\mathbb{S}=\left(\kappa+\left|\mathbb{D}_{x} \mathbf{v}\right|^{2}\right)^{\frac{q-2}{2}} \mathbb{D}_{x} \mathbf{v} \text { a.e. in }(0, T) \times \Omega ;
$$

(iv) it holds the weak formulation

$$
\begin{gathered}
\int_{0}^{T}\left\langle\partial_{t} \mathbf{v}, \boldsymbol{\varphi}\right\rangle \mathrm{d} t-\int_{0}^{T} \int_{\Omega} \mathbf{v} \otimes \mathbf{v}: \nabla_{x} \varphi \mathrm{d} x \mathrm{~d} t+\int_{0}^{T} \int_{\Omega} \mathbb{S}: \mathbb{D}_{x} \boldsymbol{\varphi} \mathrm{d} x \mathrm{~d} t \\
=\int_{0}^{T} \int_{\Omega} \mathbf{b} \cdot \boldsymbol{\varphi} \mathrm{d} x \mathrm{~d} t \text { for any } \boldsymbol{\varphi} \in C^{\infty}\left((0, T) ; C_{0, \text { div }}^{\infty}\left(\Omega ; R^{3}\right)\right) ;
\end{gathered}
$$

(v) $\mathbf{v}(T, \cdot)=\mathbf{v}(0, \cdot)$ in $L^{2}\left(\Omega ; R^{3}\right)$.

We state the main result.

Theorem 1.3. Let $\Omega \subset R^{3}$ a domain, let $T>0, \kappa \geq 0$ and let $q>6 / 5$. Assume $\mathbf{b} \in C\left([0, T] ; L^{2}\left(\Omega ; R^{3}\right)\right)$ and that is a time-periodic function with period $T$. Then there exists a time-periodic weak solution $\mathbf{v}$ with period $T$ to system (1.1).

Remark 1.4. From the proof given in Sect. 2 we may infer that the result stated in Theorem 1.3 holds true for more complicated models than $(1.1 \mathrm{c})$ and in particular belonging to the class $\mathbb{G}(\mathbb{S}, \mathbb{D})=0$ where $\mathbb{G}$ describes a $q$-graph, see [6, Appendix B].

The property of extinction in a finite time of the solutions to a $q$-parabolic system when $q \in(1,2)$ is well known, see $[10,12]$ and the same is true for a q-Stokes problem $[2,9]$. In the following theorem we prove that in the case $q \in(6 / 5,2)$ the system (1.1) admits a time-periodic weak solution which periodically becomes extinct.

Corollary 1.5. Let $\Omega \subset R^{3}$ a domain, let $T>0, \kappa=0$ and let $q \in(6 / 5,2)$. Assume that $\mathbf{b} \in$ $C\left([0, T] ; L^{2}\left(\Omega ; R^{3}\right)\right)$ is a time-periodic function with period $T$ and that admits an extinction instant $\bar{t} \in(0, T)$, meaning that

$$
\|\mathbf{b}(t)\|_{L^{2}\left(\Omega ; R^{3}\right)}=0 \text { for a.a. } t \in(\bar{t}, T),
$$

and $\bar{t}+\frac{\bar{K}^{2-q}}{\alpha(2-q)} \leq T$ where

$$
\bar{K}=\left(\frac{C_{2}\left(\max _{[0, T]}\|\mathbf{b}\|_{L^{2}\left(\Omega_{T} ; R^{3}\right)}^{q^{\prime}}+1\right)}{C_{1} C_{S}}\right)^{\frac{1}{q}}
$$

and $C_{1}, C_{2}, C_{S}, \alpha$ are positive constant depending on $q$ and $\Omega$. Then there exists a time-periodic weak solution $\mathbf{v}$ with period $T$ to system (1.1) such that admits an extinction instant:

$$
\|\mathbf{v}(t)\|_{L^{2}\left(\Omega ; R^{3}\right)}=0 \text { for a.a. } t \in\left(\bar{t}_{v}, T\right)
$$

with $\bar{t}_{v} \leq \bar{t}+\frac{\bar{K}^{2-q}}{\alpha(2-q)}$. 
Remark 1.6. The study of the existence of an instant of extinction for $\mathbf{v}$ is only qualitative, for such reason it might be $\|\mathbf{v}(t)\|_{L^{2}\left(\Omega ; R^{3}\right)}=0$ for a.a. $t \in\left(\bar{t}_{v}, T\right)$ with

$$
\bar{t}_{v} \leq \bar{t}+\frac{\bar{K}^{2-q}}{\alpha(2-q)} .
$$

Notation and function spaces Let $\Omega \subset R^{d}$ a domain, i.e. an open bounded connected set. Throughout the paper we fix the dimension $d=3$, however we introduce the notation for a general dimension $d$. We denote the standard Lebesgue and Sobolev spaces of scalar functions by the usual notation $\left(L^{r}(\Omega),\|\cdot\|_{L^{r}(\Omega)}\right)$ and $\left(W^{k, r}(\Omega),\|\cdot\|_{W^{k, r}(\Omega)}\right)$. For vector-valued or tensor-valued functions, i.e. with value in $R^{d}$ or $R^{d \times d}$ respectively, we denote the corresponding Lebesgue and Sobolev spaces by $\left(L^{r}\left(\Omega ; R^{d}\right),\|\cdot\|_{L^{r}\left(\Omega ; R^{d}\right)}\right)$ and $\left(W^{k, r}\left(\Omega ; R^{d \times d}\right),\|\cdot\|_{W^{k, r}\left(\Omega ; R^{d \times d}\right)}\right)$ (with $1 \leq r \leq+\infty$ and $\left.k \in N\right)$. If $\left(X,\|\cdot\|_{X}\right)$ is a Banach space, $X^{*}$ is its dual space; then $L^{r}((0, T) ; X)$ is the relevant Bochner space, while $C([0, T] ; X)$ is the space of continuous functions from $[0, T]$ with values in $X$ and $C_{\text {weak }}([0, T] ; X)$ is the space of weakly continuous functions from $[0, T]$ with values in $X$. Next, we define the space of compactly supported smooth functions and its subspace of solenoidal functions:

$$
\begin{aligned}
C_{0}^{\infty}\left(\Omega ; R^{d}\right) & :=\left\{\mathbf{u}: \Omega \rightarrow R^{d}, \mathbf{u} \text { smooth, supp } \mathbf{u} \subset \Omega\right\}, \\
C_{0, \operatorname{div}}^{\infty}\left(\Omega ; R^{d}\right) & :=\left\{\mathbf{u} \in C_{0}^{\infty}\left(\Omega ; R^{d}\right), \operatorname{div}_{x} \mathbf{u}=0\right\},
\end{aligned}
$$

and their closures in $W^{k, r}$-norm for any $1<r<+\infty$ and $k \in N$ :

$$
W_{0}^{k, r}\left(\Omega ; R^{d}\right):={\overline{C_{0}^{\infty}\left(\Omega ; R^{d}\right)}}^{\|\cdot\|_{W^{k, r}}}, W_{0, \mathrm{div}}^{k, r}\left(\Omega ; R^{d}\right):={\overline{C_{0, \operatorname{div}}^{\infty}\left(\Omega ; R^{d}\right)}}^{\|\cdot\|_{W^{k, r}}} .
$$

Note that for a domain $\Omega$ (without further regularity assumption on the smoothness of $\partial \Omega$ ) we have the embedding $W_{0, \text { div }}^{3,2}\left(\Omega ; R^{3}\right) \hookrightarrow W_{0}^{3,2}\left(\Omega ; R^{3}\right) \hookrightarrow W^{1, \infty}\left(\Omega ; R^{3}\right)$. (We will consider in particular the space $W_{0, \text { div }}^{3,2}\left(\Omega ; R^{3}\right)$ for the basis for the Galerkin method.)

As a consequence of the Poincaré and Korn inequalities the following norm are equivalent on the spaces $W_{0}^{1, r}$ and $W_{0, \text { div }}^{1, r}$ for any $1<r<\infty$ :

$$
\left\|\mathbb{D}_{x} \boldsymbol{\varphi}\right\|_{L^{r}\left(\Omega ; R^{d}\right)} \leq\left\|\nabla_{x} \boldsymbol{\varphi}\right\|_{L^{r}\left(\Omega ; R^{d}\right)} \leq\|\boldsymbol{\varphi}\|_{W^{1, r}\left(\Omega ; R^{d}\right)} \leq C_{P} C_{K}\left\|\mathbb{D}_{x} \boldsymbol{\varphi}\right\|_{L^{r}\left(\Omega ; R^{d}\right)}
$$

for all $\varphi \in W^{1, r}\left(\Omega ; R^{d}\right)$, where the constant $C_{P}>0$ that appears due to the Poincaré inequality depends on $r$ and $\Omega$, while the constant $C_{K}>0$ that appears due to the Korn inequality depends only on $r$.

Finally, for any vector valued function $\varphi$ the symmetric part of the gradient is defined by $\mathbb{D}_{x} \varphi:=$ $\frac{1}{2}\left(\nabla_{x} \boldsymbol{\varphi}+\left(\nabla_{x} \boldsymbol{\varphi}\right)^{t}\right)$.

\section{Proof of Theorem 1.3}

\subsection{The Case $q \geq \frac{11}{5}$}

Existence of approximations For any $\varepsilon>0$, let us introduce the approximating system

$$
\begin{aligned}
\partial_{t} \mathbf{v}+\operatorname{div}_{x}(\mathbf{v} \otimes \mathbf{v})-\operatorname{div}_{x} \mathbb{S}+\nabla_{x} p-\varepsilon \Delta_{x} \mathbf{v} & =\mathbf{b} \text { in }(0, T) \times \Omega, \\
\operatorname{div}_{x} \mathbf{v} & =0 \text { in }(0, T) \times \Omega, \\
\mathbf{v} & =0 \text { on }(0, T) \times \partial \Omega .
\end{aligned}
$$

where

$$
\mathbb{S}=\left|\mathbb{D}_{x} \mathbf{v}\right|^{q-2} \mathbb{D}_{x} \mathbf{v}
$$

Let us remark that in this paragraph we perform the proof for the rheological law stated above where the parameter $\kappa=0$, but through the same steps the result follows in the case $\kappa>0$. 
In order to fix the basis for the Galerkin method, consider the eigenvalue problem

$$
((\boldsymbol{\omega}, \boldsymbol{\varphi}))=\lambda(\boldsymbol{\omega}, \boldsymbol{\varphi}) \text { for all } \boldsymbol{\varphi} \in W_{0, \mathrm{div}}^{3,2}\left(\Omega ; R^{3}\right)
$$

with $\lambda \in R$ and $\boldsymbol{\omega} \in W_{0, \text { div }}^{3,2}\left(\Omega ; R^{3}\right)$, and where $(\cdot, \cdot)$ is the scalar product in $L^{2}\left(\Omega ; R^{3}\right)$ while $((\cdot, \cdot))$ is the scalar product in $W_{0, \text { div }}^{3,2}\left(\Omega ; R^{3}\right)$ defined as $((\boldsymbol{\omega}, \boldsymbol{\varphi})):=\left(\nabla^{3} \boldsymbol{\omega}, \nabla^{3} \boldsymbol{\varphi}\right)+(\boldsymbol{\omega}, \boldsymbol{\varphi})$. It is known (see e.g. [20, Appendix A.4]) that there exist eigenvalues $\left\{\lambda_{m}\right\}_{m \in N}$ and corresponding eigenfunctions $\left\{\boldsymbol{\omega}^{m}\right\}_{m \in N}$ such that they are orthonormal in $L^{2}\left(\Omega, R^{3}\right)$ and orthogonal in $W_{0 \text {,div }}^{3,2}$. For any fixed $n \in N$ the projectors $P^{n}: W_{0, \text { div }}^{3,2} \rightarrow X_{n}:=\operatorname{span}\left\{\boldsymbol{\omega}^{1}, \ldots, \boldsymbol{\omega}^{n}\right\}$ defined as $P^{n} \mathbf{v}:=\sum_{i=1}^{n}\left(\mathbf{v}, \boldsymbol{\omega}^{i}\right) \boldsymbol{\omega}^{i}$ are continuous orthonormal projectors in $L^{2}\left(\Omega, R^{3}\right)$. It is worth recalling that $W_{0, \text { div }}^{3,2}\left(\Omega ; R^{3}\right) \hookrightarrow W_{0}^{3,2}\left(\Omega ; R^{3}\right) \hookrightarrow W^{1, \infty}\left(\Omega, R^{3}\right)$.

Now, given $\mathbf{v}_{0}^{n} \in \operatorname{span}\left\{\boldsymbol{\omega}^{1}, \ldots, \boldsymbol{\omega}^{n}\right\}$ with

$$
\left\|\mathbf{v}_{0}^{n}\right\|_{L^{2}\left(\Omega ; R^{3}\right)} \leq K
$$

with $K$ constant defined as

$$
K:=\left(\frac{C_{2} \max _{[0, T]}\|\mathbf{b}\|_{L^{2}\left(\Omega ; R^{3}\right)}^{q^{\prime}}}{C_{1} C_{S}}\right)^{\frac{1}{q}}
$$

(with constants $C_{1}, C_{2}, C_{S}$ defined later in the proof, also the reasons will be understood), we look for the Galerkin approximation

$$
\mathbf{v}^{n}(t, x):=\sum_{i=1}^{n} c_{i}^{n}(t) \boldsymbol{\omega}^{i}(x)
$$

such that it satisfies for any $k=1, \ldots, n$

$$
\left(\partial_{t} \mathbf{v}^{n}, \vec{\omega}^{k}\right)-\left(\mathbf{v}^{n} \otimes \mathbf{v}^{n}, \nabla_{x} \boldsymbol{\omega}^{k}\right)+\left(\mathbb{S}^{n}, \mathbb{D}_{x} \boldsymbol{\omega}^{k}\right)+\varepsilon\left(\nabla_{x} \mathbf{v}^{n}, \nabla_{x} \boldsymbol{\omega}^{k}\right)=\left(\mathbf{b}, \boldsymbol{\omega}^{k}\right)
$$

where

$$
\mathbb{S}^{n}:=\left|\mathbb{D}_{x} \mathbf{v}^{n}\right|^{q-2} \mathbb{D}_{x} \mathbf{v}^{n}
$$

and

$$
\mathbf{v}^{n}(0)=\mathbf{v}_{0}^{n},
$$

meaning in components that for any $k=1, \ldots, n$

$$
\begin{aligned}
\left(c_{k}^{n}(t)\right)^{\prime} & =\sum_{i, j=1}^{n} c_{i}^{n} c_{j}^{n} f_{i j k}-\left(\mathbb{S}^{n}, \mathbb{D}_{x} \boldsymbol{\omega}^{k}\right)-\varepsilon \sum_{i=1}^{n} c_{i}^{n} g_{i k}+b_{k}, \\
c_{k}^{n}(0) & =c_{0, k}^{n}:=\left(\mathbf{v}_{0}^{n}, \boldsymbol{\omega}^{k}\right),
\end{aligned}
$$

where we used the notation

$$
f_{i j k}:=\left(\boldsymbol{\omega}^{i} \otimes \boldsymbol{\omega}^{j}, \nabla_{x} \boldsymbol{\omega}^{k}\right), g_{i k}:=\left(\nabla_{x} \boldsymbol{\omega}^{i}, \nabla_{x} \boldsymbol{\omega}^{k}\right), b_{k}:=\left(\mathbf{b}, \boldsymbol{\omega}^{k}\right) .
$$

The existence and uniqueness of the solution $\mathbf{c}^{n}(t)=\left(c_{1}^{n}(t), \ldots, c_{n}^{n}(t)\right)$ to the Cauchy problem $(2.5)$ in a local time interval $\left[0, t_{n}\right)$ follow by standard results on ODEs being the right-hand side of $(2.5 \mathrm{a})$ a Lipschitz function thanks to the assumption $q \geq 11 / 5$.

Multiplying (2.4a) by $c_{k}^{n}$ and summing over $k=1, \ldots, n$, then employing the Korn and the Young inequalities we get

$$
\begin{array}{r}
\frac{d}{d t}\left\|\mathbf{v}^{n}(t)\right\|_{L^{2}\left(\Omega ; R^{3}\right)}^{2}+C_{1}\left\|\mathbb{D}_{x} \mathbf{v}^{n}\right\|_{L^{q}\left(\Omega ; R^{3 \times 3}\right)}^{q}+\varepsilon\left\|\nabla_{x} \mathbf{v}^{n}\right\|_{L^{2}\left(\Omega ; R^{3 \times 3}\right)}^{2} \\
\leq C_{2}\|\mathbf{b}\|_{L^{\prime}\left(\Omega ; R^{3}\right)}^{q^{\prime}}
\end{array}
$$

then integrating in time over $(0, t)$ we obtain

$$
\left\|\mathbf{v}^{n}(t)\right\|_{L^{2}\left(\Omega ; R^{3}\right)}^{2} \leq\left\|\mathbf{v}_{0}^{n}\right\|_{L^{2}\left(\Omega ; R^{3}\right)}^{2}+C_{2} T \max _{[0, T]}\|\mathbf{b}\|_{L^{2}\left(\Omega ; R^{3}\right)}^{q^{\prime}} \text { for all } t \in[0, T] .
$$


Note that $\left\|\mathbf{v}^{n}(t)\right\|_{L^{2}\left(\Omega ; R^{3}\right)}^{2}=\left|\mathbf{c}^{n}(t)\right|^{2}$, thus $\mathbf{v}^{n}(t)$ is well-defined on the whole interval $[0, T]$ and $\mathbf{v}^{n} \in$ $C\left([0, T] ; L^{2}\left(\Omega ; R^{3}\right)\right)$.

Existence of a periodic in time approximating solution Now, by (2.6) and by the assumption in (2.3) we realize that

$$
\left|\mathbf{c}^{n}(t)\right|=\left\|\mathbf{v}^{n}(t)\right\|_{L^{2}\left(\Omega ; R^{3}\right)} \leq K \text { for all } t \in[0, T],
$$

indeed if there exists an instant $\bar{t}$ such that $\left\|\mathbf{v}^{n}(\bar{t})\right\|_{2}=K$ then

$$
\frac{d}{d t}\left\|\mathbf{v}^{n}(\bar{t})\right\|_{L^{2}\left(\Omega ; R^{3}\right)}^{2}<C_{2} \max _{[0, T]}\|\mathbf{b}\|_{L^{2}\left(\Omega ; R^{3}\right)}^{q^{\prime}}-C_{1} C_{S} K^{q}=0
$$

where $C_{S}$ is the constant due to the embedding $W_{0}^{1, q} \hookrightarrow L^{2}$ and to the Korn inequality. Being $\mathbf{v}^{n} \in$ $C\left(0, T ; L^{2}\left(\Omega ; R^{3}\right)\right),(2.9)$ implies that $\left\|\mathbf{v}^{n}(t)\right\|_{L^{2}\left(\Omega ; R^{3}\right)}^{2}$ is a non-increasing function whenever $\left\|\mathbf{v}^{n}(t)\right\|_{L^{2}\left(\Omega ; R^{3}\right)}$ $=K$, thus $(2.8)$ holds.

Consider $\mathbf{c}^{n}(t)=\left(c_{1}^{n}(t), \ldots, c_{n}^{n}(t)\right)$ and $\mathbf{d}^{n}(t)=\left(d_{1}^{n}(t), \ldots, d_{n}^{n}(t)\right)$ solutions of $(2.5 \mathrm{a})$ corresponding to the same force $\mathbf{b}=\mathbf{b}(t, x)$ and set $\alpha_{k}^{n}(t):=c_{k}^{n}(t)-d_{k}^{n}(t)$ for any $k=1, \ldots, n$. It holds for any $k=1, \ldots, n$

$$
\left(\alpha_{k}^{n}(t)\right)^{\prime}-\sum_{i, j=1}^{n}\left(\alpha_{i}^{n} c_{j}^{n}+d_{i}^{n} \alpha_{j}^{n}\right) f_{i j k}+\left(\mathbb{S}_{1}^{n}-\mathbb{S}_{2}^{n}, \mathbb{D}_{x} \boldsymbol{\omega}^{k}\right)+\varepsilon \sum_{i=1}^{n} \alpha_{i}^{n} g_{i k}=0
$$

where

$$
\mathbb{S}_{1}^{n}:=\sum_{i=1}^{n} c_{i}^{n}\left|\sum_{j=1}^{n} c_{j}^{n} \mathbb{D}_{x} \boldsymbol{\omega}^{j}\right|^{q-2} \mathbb{D}_{x} \boldsymbol{\omega}^{i} \text { and } \mathbb{S}_{2}^{n}:=\sum_{i=1}^{n} d_{i}^{n}\left|\sum_{j=1}^{n} d_{j}^{n} \mathbb{D}_{x} \boldsymbol{\omega}^{j}\right|^{q-2} \mathbb{D}_{x} \boldsymbol{\omega}^{i} .
$$

Multiplying by $\alpha_{k}^{n}$, summing over $k=1, \ldots, n$, employing the monotonicity of the operator in (1.1c), the Poincaré inequality and the fact that

$$
\sum_{i, j, k=1}^{n} \alpha_{i}^{n}(t) c_{j}^{n}(t) \alpha_{k}^{n}(t) f_{i j k}=0
$$

we get

$$
\begin{array}{r}
\frac{1}{2} \frac{d}{d t}\left\|\mathbf{u}^{n}(t)\right\|_{L^{2}\left(\Omega ; R^{3}\right)}^{2}-\sum_{i, j, k=1}^{n} d_{i}^{n}(t) \alpha_{j}^{n}(t) \alpha_{k}^{n}(t) f_{i j k} \\
+\varepsilon C_{3}\left\|\mathbf{u}^{n}(t)\right\|_{L^{2}\left(\Omega ; R^{3}\right)}^{2} \leq 0
\end{array}
$$

where

$$
\mathbf{u}^{n}(t):=\sum_{k=1}^{n} \alpha_{k}^{n}(t) \boldsymbol{\omega}^{k} .
$$

Now, we follow the idea in [24] to treat the convective term. Employing the regularity of the basis functions $\boldsymbol{\omega}^{i}$, for any $n \in N$ it holds that

$$
\begin{aligned}
& \left|\sum_{i, j, k=1}^{n} \alpha_{j}^{n}(t) d_{i}^{n}(t) \alpha_{k}^{n}(t) f_{i j k}\right| \leq n \max _{i, j, k} f_{i j k} \max _{[0, T]}\left|\mathbf{d}^{n}(t)\right| \sum_{j, k=1}^{n}\left|\alpha_{j}^{n}(t)\right|\left|\alpha_{k}^{n}(t)\right| \\
& \leq n^{2} \max _{i, j, k} f_{i j k} \max _{[0, T]}\left|\mathbf{d}^{n}(t)\right|\left(\sum_{j=1}^{n} \frac{1}{2}\left|\alpha_{j}^{n}(t)\right|^{2}+\sum_{k=1}^{n} \frac{1}{2}\left|\alpha_{k}^{n}(t)\right|^{2}\right) \\
& =C_{4}\left\|\mathbf{u}^{n}(t)\right\|_{L^{2}\left(\Omega ; R^{3}\right)}^{2}
\end{aligned}
$$

where we set $C_{4}:=n^{2} \max _{i, j, k} f_{i j k} \max _{[0, T]}\left|\mathbf{d}^{n}(t)\right|$. Employing (2.12) in (2.11), we obtain

$$
\frac{d}{d t}\left\|\mathbf{u}^{n}(t)\right\|_{L^{2}\left(\Omega ; R^{3}\right)} \leq\left(C_{4}-\varepsilon C_{3}\right)\left\|\mathbf{u}^{n}(t)\right\|_{L^{2}\left(\Omega ; R^{3}\right)},
$$


which implies

$$
\left\|\mathbf{u}^{n}(t)\right\|_{L^{2}\left(\Omega ; R^{3}\right)} \leq e^{\left(C_{4}-\varepsilon C_{3}\right) t}\left\|\mathbf{u}^{n}(0)\right\|_{L^{2}\left(\Omega ; R^{3}\right)} \text { for any } t \in[0, T]
$$

and it means that

$$
\left\|\mathbf{v}^{n}(t)-\mathbf{z}^{n}(t)\right\|_{L^{2}\left(\Omega ; R^{3}\right)} \leq e^{\left(C_{4}-\varepsilon C_{3}\right) t}\left\|\mathbf{v}^{n}(0)-\mathbf{z}^{n}(0)\right\|_{L^{2}\left(\Omega ; R^{3}\right)}
$$

for any $t \in[0, T]$, where $\mathbf{v}^{n}(t):=\sum_{k=1}^{n} c_{k}^{n}(t) \boldsymbol{\omega}^{k}$ and $\mathbf{z}^{n}(t):=\sum_{k=1}^{n} d_{k}^{n}(t) \boldsymbol{\omega}^{k}$. Let us consider the mapping

$$
F: R^{n} \rightarrow R^{n}, F\left(\mathbf{c}_{0}^{n}\right):=\mathbf{c}^{n}(T)
$$

where $\mathbf{c}^{n}(t)$ is the solution to the system (2.5) corresponding to the initial value $\mathbf{c}_{0}^{n}$. The mapping $F$ is well-defined as the solution to (2.5) there exists and it is unique. Moreover, $F$ transforms the ball $B_{K}$ of $R^{n}$ into itself by (2.8) and it is continuous by (2.15). Then the Brouwer theorem ensures the existence of a fixed point, hence for any $n \in N$ there exists $\mathbf{v}^{n}(t)$ solution to (2.4a) such that

$$
\mathbf{v}^{n}(T)=\mathbf{v}^{n}(0) .
$$

Limit as $n \rightarrow+\infty$. Integrating (2.6) in time over $(0, T)$ we have

$$
\begin{aligned}
& \sup _{n} \max _{t \in[0, T]}\left\|\mathbf{v}^{n}(t)\right\|_{L^{2}\left(\Omega ; R^{3}\right)} \\
& \quad+\sup _{n}\left(\left\|\mathbb{D}_{x} \mathbf{v}^{n}\right\|_{L^{q}\left(\Omega_{T} ; R^{3 \times 3}\right)}+\varepsilon\left\|\nabla_{x} \mathbf{v}^{n}\right\|_{L^{2}\left(\Omega_{T} ; R^{3 \times 3}\right)}\right)<+\infty
\end{aligned}
$$

where we introduced the notation $\Omega_{T}:=(0, T) \times \Omega$, and as a consequence

$$
\sup _{n}\left\|\mathbb{S}^{n}\right\|_{L^{q^{\prime}}\left(\Omega_{T} ; R^{3 \times 3}\right)}<+\infty .
$$

From the interpolation inequality

$$
\int_{\Omega}\left|\mathbf{v}^{n}\right|^{\frac{5 q}{3}} \mathrm{~d} x \leq\left(\int_{\Omega}\left|\mathbf{v}^{n}\right|^{2} \mathrm{~d} x\right)^{\frac{q}{3}}\left(\int_{\Omega}\left|\mathbf{v}^{n}\right|^{\frac{3 q}{3-q}} \mathrm{~d} x\right)^{\frac{3-q}{3}},
$$

integrating in time and using Sobolev embeddings, we achieve that

$$
\int_{0}^{T} \int_{\Omega}\left|\mathbf{v}^{n}\right|^{\frac{5 q}{3}} \mathrm{~d} x \mathrm{~d} t \leq \sup _{[0, T]}\left\|\mathbf{v}^{n}\right\|_{L^{2}\left(\Omega ; R^{3}\right)}^{\frac{2 q}{3}} \int_{0}^{T} \int_{\Omega}\left|\nabla_{x} \mathbf{v}^{n}\right|^{q} \mathrm{~d} x \mathrm{~d} t,
$$

next (2.16) and (2.7) together with the Korn inequality imply that

$$
\sup _{n}\left\|\mathbf{v}^{n}\right\|_{L^{\frac{5 q}{3}}\left(\Omega_{T} ; R^{3}\right)}<+\infty .
$$

For any $\varphi \in L^{q}\left(0, T ; W_{0, \operatorname{div}}^{3,2}\left(\Omega ; R^{3}\right)\right)$

$$
\int_{0}^{T} \int_{\Omega} \partial_{t} \mathbf{v}^{n} \cdot \boldsymbol{\varphi} \mathrm{d} x \mathrm{~d} t=\int_{0}^{T} \int_{\Omega} \partial_{t} \mathbf{v}^{n} \cdot P^{n} \boldsymbol{\varphi} \mathrm{d} x \mathrm{~d} t
$$

then from (2.4a) using (2.16), (2.17), (2.19) and the continuity of $P^{n}$ we obtain that

$$
\sup _{n}\left\|\partial_{t} \mathbf{v}^{n}\right\|_{\left(L^{q}\left(0, T ; W_{0, \text { div }}^{3,2}\left(\Omega ; R^{3}\right)\right)\right)^{*}}<+\infty .
$$

Note that it suffices to take $\varphi \in L^{q}\left(0, T ; W_{0, \text { div }}^{3,2}\left(\Omega ; R^{3}\right)\right)$ since it coincides with the intersection of the spaces $L^{q}\left(0, T ; W_{0, \operatorname{div}}^{3,2}\left(\Omega ; R^{3}\right)\right), L^{q}\left(0, T ; W_{0, \operatorname{div}}^{1, q}\left(\Omega ; R^{3}\right)\right)$ and $L^{\frac{5 q}{5 q-6}}\left(0, T ; W_{0, \operatorname{div}}^{1, \frac{5 q}{5 q-6}}\left(\Omega ; R^{3}\right)\right)$ being $q \geq 11 / 5$. 
For any fixed $\varepsilon>0$ and for any $n \in N$ the established estimates uniform respect to $n$ yield the existence of $\mathbf{v}$ and $\mathbb{S}$ such that the following convergences hold (for suitable subsequences not relabelled):

$$
\begin{aligned}
\mathbf{v}^{n} & \rightarrow \mathbf{v} & & \text { weakly-* in } L^{\infty}\left(0, T ; L^{2}\left(\Omega ; R^{3}\right)\right), \\
\mathbf{v}^{n} & \rightarrow \mathbf{v} & & \text { weakly in } L^{q}\left(0, T ; W_{0, \text { div }}^{1, q}\left(\Omega ; R^{3}\right)\right), \\
\partial_{t} \mathbf{v}^{n} & \rightarrow \partial_{t} \mathbf{v} & & \text { weakly in }\left(L^{q}\left(0, T ; W_{0, \text { div }}^{3,2}\left(\Omega ; R^{3}\right)\right)\right)^{*}, \\
\mathbb{S}^{n} & \rightarrow \mathbb{S} & & \text { weakly in } L^{q^{\prime}}\left(\Omega_{T} ; R^{3 \times 3}\right), \\
\nabla_{x} \mathbf{v}^{n} & \rightarrow \nabla_{x} \mathbf{v} & & \text { weakly in } L^{2}\left(\Omega_{T} ; R^{3 \times 3}\right) .
\end{aligned}
$$

Furthermore, using (2.22) and (2.23), the Aubin-Lions lemma applied to $W_{0, \operatorname{div}}^{3,2} \hookrightarrow W_{0}^{1, q} \hookrightarrow \hookrightarrow L^{q} \hookrightarrow$ $\left(W_{0, \text { div }}^{3,2}\right)^{*}$ gives

$$
\mathbf{v}^{n} \rightarrow \mathbf{v} \text { strongly in } L^{q}\left(\Omega_{T} ; R^{3}\right),
$$

which together with (2.19) and the following interpolation inequality

$$
\begin{aligned}
\left\|\mathbf{v}^{n}-\mathbf{v}\right\|_{r} & \leq\left\|\mathbf{v}^{n}-\mathbf{v}\right\|_{q}^{a}\left\|\mathbf{v}^{n}-\mathbf{v}\right\|_{\frac{5 q}{3}}^{1-a} \\
& \leq C\left\|\mathbf{v}^{n}-\mathbf{v}\right\|_{q}^{a} \text { for any } r<\frac{5 q}{3}, a \in(0,1),
\end{aligned}
$$

implies

$$
\mathbf{v}^{n} \rightarrow \mathbf{v} \text { strongly in } L^{r}\left(\Omega_{T} ; R^{3}\right) \text { for any } r<\frac{5 q}{3} .
$$

It is worth noting that, (2.22), (2.23) ensure

$$
\mathbf{v} \in C\left([0, T] ; L^{2}\left(\Omega ; R^{3}\right)\right) .
$$

From the bound in (2.8) we obtain that for any $\varphi \in C_{0}^{\infty}\left(\Omega ; R^{3}\right)$

$$
\left(\mathbf{v}^{n}(T), \varphi\right) \rightarrow(\mathbf{v}(T), \varphi) \text { as } n \rightarrow+\infty .
$$

On the other side, by (2.3) we get the existence of a subsequence $\mathbf{v}_{0}^{n}$ such that

$$
\mathbf{v}_{0}^{n} \rightarrow \mathbf{v}_{0} \text { weakly in } L^{2}\left(\Omega ; R^{3}\right)
$$

with $\mathbf{v}_{0}$ satisfying again the bound (2.3). Therefore, employing (2.29) and (2.30) it follows

$$
\left(\mathbf{v}(T)-\mathbf{v}_{0}, \boldsymbol{\varphi}\right)=\lim _{n \rightarrow+\infty}\left(\mathbf{v}^{n}(T)-\mathbf{v}_{0}^{n}, \boldsymbol{\varphi}\right)=0
$$

i.e. the limit function $\mathbf{v}$ is time-periodic with period $T$. Finally thanks to the convergences $(2.21)-(2.27)$, using that $W_{0, \text { div }}^{3,2}$ is dense in $W_{0, \text { div }}^{1, q}$ for any $q \geq 1$, and employing a standard density argument we achieve that $\mathbf{v}$ and $\mathbb{S}$ enjoy the following weak formulation:

$$
\begin{aligned}
\int_{0}^{T} & \left\langle\partial_{t} \mathbf{v}, \boldsymbol{\varphi}\right\rangle \mathrm{d} t+\int_{0}^{T} \int_{\Omega} \mathbb{S}: \mathbb{D}_{x} \varphi \mathrm{d} x \mathrm{~d} t+\varepsilon \int_{0}^{T} \int_{\Omega} \nabla_{x} \mathbf{v}: \nabla_{x} \varphi \mathrm{d} x \mathrm{~d} t \\
= & \int_{0}^{T} \int_{\Omega} \mathbf{v} \otimes \mathbf{v}: \nabla_{x} \boldsymbol{\varphi} \mathrm{d} x \mathrm{~d} t+\int_{0}^{T} \int_{\Omega} \mathbf{b} \cdot \boldsymbol{\varphi} \mathrm{d} x \mathrm{~d} t
\end{aligned}
$$

for any $\varphi \in L^{q}\left(0, T ; W_{0, \text { div }}^{1, q}\left(\Omega ; R^{3}\right)\right)$. Thus $\partial_{t} \mathbf{v} \in\left(L^{q}\left(0, T ; W_{0, \text { div }}^{1, q}\left(\Omega ; R^{3}\right)\right)\right)^{*}$. It remains to show that $\mathbb{S}$ fulfils (1.1c). Multiplying (2.4a) by $c_{k}^{n}$, summing over $k=1, \ldots, n$ and integrating in time over $(0, T)$ we have

$$
\int_{0}^{T} \int_{\Omega} \mathbb{S}^{n}: \mathbb{D}_{x} \mathbf{v}^{n} \mathrm{~d} x \mathrm{~d} t+\varepsilon \int_{0}^{T} \int_{\Omega}\left|\nabla_{x} \mathbf{v}^{n}\right|^{2} \mathrm{~d} x \mathrm{~d} t=\int_{0}^{T} \int_{\Omega} \mathbf{b} \cdot \mathbf{v}^{n} \mathrm{~d} x \mathrm{~d} t,
$$

taking the limsup and employing (2.22) and (2.25) it follows

$$
\limsup _{n \rightarrow+\infty} \int_{0}^{T} \int_{\Omega} \mathbb{S}^{n}: \mathbb{D}_{x} \mathbf{v}^{n} \mathrm{~d} x \mathrm{~d} t \leq-\varepsilon \int_{0}^{T}\left\|\nabla_{x} \mathbf{v}\right\|_{2}^{2} \mathrm{~d} t+\int_{0}^{T} \int_{\Omega} \mathbf{b} \cdot \mathbf{v} \mathrm{d} x \mathrm{~d} t .
$$


Comparing (2.33) with the outcome of (2.32) choosing $\varphi=\mathbf{v}$ it follows

$$
\limsup _{n \rightarrow+\infty} \int_{0}^{T} \int_{\Omega} \mathbb{S}^{n}: \mathbb{D}_{x} \mathbf{v}^{n} \mathrm{~d} x \mathrm{~d} t \leq \int_{0}^{T} \int_{\Omega} \mathbb{S}: \mathbb{D}_{x} \mathbf{v} \mathrm{d} x \mathrm{~d} t
$$

and finally by virtue of the established convergences together with (2.34) the standard Minty's trick ensures that

$$
\mathbb{S}=|\mathbb{D} \mathbf{v}|^{q-2} \mathbb{D} \mathbf{v} \text { a.e. in } \Omega_{T} .
$$

Limit as $\varepsilon \rightarrow 0$. For any $\varepsilon>0$ we constructed $\mathbf{v}^{\varepsilon}$ and $\mathbb{S}^{\varepsilon}$ fulfilling

$$
\begin{aligned}
\int_{0}^{T} & \left\langle\partial_{t} \mathbf{v}^{\varepsilon}, \boldsymbol{\varphi}\right\rangle \mathrm{d} t+\int_{0}^{T} \int_{\Omega} \mathbb{S}^{\varepsilon}: \mathbb{D}_{x} \boldsymbol{\varphi} \mathrm{d} x \mathrm{~d} t+\varepsilon \int_{0}^{T} \int_{\Omega} \nabla_{x} \mathbf{v}^{\varepsilon}: \nabla_{x} \boldsymbol{\varphi} \mathrm{d} x \mathrm{~d} t \\
= & \int_{0}^{T} \int_{\Omega} \mathbf{v}^{\varepsilon} \otimes \mathbf{v}^{\varepsilon}: \nabla_{x} \boldsymbol{\varphi} \mathrm{d} x \mathrm{~d} t+\int_{0}^{T} \int_{\Omega} \mathbf{b} \cdot \boldsymbol{\varphi} \mathrm{d} x \mathrm{~d} t
\end{aligned}
$$

for any $\varphi \in L^{q}\left(0, T ; W_{0, \text { div }}^{1, q}\left(\Omega ; R^{3}\right)\right)$, with $\mathbf{v}^{\varepsilon}$ time-periodic with period $T$. Taking $\mathbf{v}^{\varepsilon}$ as test function we derive uniform estimates that ensure the following convergences as $\varepsilon \rightarrow 0$ (for suitable subsequences not relabelled):

$$
\begin{array}{rlrl}
\mathbf{v}^{\varepsilon} & \rightarrow \mathbf{v} & \text { weakly-* in } L^{\infty}\left(0, T ; L^{2}\left(\Omega ; R^{3}\right)\right), \\
\mathbf{v}^{\varepsilon} \rightarrow \mathbf{v} & \text { weakly in } L^{q}\left(0, T ; W_{0}^{1, q}\left(\Omega ; R^{3}\right)\right), \\
\partial_{t} \mathbf{v}^{\varepsilon} \rightarrow \partial_{t} \mathbf{v} & \text { weakly in }\left(L^{q}\left(0, T ; W_{0}^{1, q}\left(\Omega ; R^{3}\right)\right)\right)^{*}, \\
\mathbf{v}^{\varepsilon} \rightarrow \mathbf{v} & \text { strongly in } L^{r}\left(\Omega_{T} ; R^{3}\right) \text { for any } r<\frac{5 q}{3}, \\
\mathbb{S}^{\varepsilon} \rightarrow \mathbb{S} & \text { weakly in } L^{q^{\prime}}\left(\Omega_{T} ; R^{3 \times 3}\right) .
\end{array}
$$

Being $5 q / 6<q,\left\|\nabla_{x} \mathbf{v}^{\varepsilon}\right\|_{\frac{5 q}{6}}$ is uniformly bounded and as a consequence it results

$$
\left|\varepsilon \int_{0}^{T} \int_{\Omega} \nabla \mathbf{v}: \nabla \varphi\right| \leq \varepsilon\left\|\nabla \mathbf{v}^{\varepsilon}\right\|_{\frac{5 q}{6}}\|\nabla \varphi\|_{\frac{5 q}{5 q-6}} \rightarrow 0 \text { as } \varepsilon \rightarrow 0 .
$$

We are in a position to take the limit as $\varepsilon \rightarrow 0$ in (2.35) and employing the convergences in (2.36)-(2.41) we obtain the existence of $(\mathbf{v}, \mathbb{S})$ such that

$$
\begin{aligned}
& \int_{0}^{T}\left\langle\partial_{t} \mathbf{v}, \boldsymbol{\varphi}\right\rangle \mathrm{d} t+\int_{0}^{T} \int_{\Omega} \mathbb{S}: \mathbb{D}_{x} \boldsymbol{\varphi} \mathrm{d} x \mathrm{~d} t=\int_{0}^{T} \int_{\Omega} \mathbf{v} \otimes \mathbf{v}: \nabla_{x} \varphi \mathrm{d} x \mathrm{~d} t \\
& \quad+\int_{0}^{T} \int_{\Omega} \mathbf{b} \cdot \boldsymbol{\varphi} \mathrm{d} x \mathrm{~d} t \text { for any } \boldsymbol{\varphi} \in L^{q}\left(0, T ; W_{0, \text { div }}^{1, q}\left(\Omega ; R^{3}\right)\right) .
\end{aligned}
$$

Employing the same tools of the previous passage to the limit as $n \rightarrow+\infty$ we obtain that $\mathbf{v}$ is timeperiodic with period $T$ and that $\mathbb{S}$ fulfils the rheological law (1.1c).

\subsection{The Case $q \in\left(\frac{6}{5}, \frac{11}{5}\right)$}

Existence of a periodic in time approximating solution For any $\varepsilon, \kappa>0$ let us introduce the approximating system

$$
\begin{aligned}
\partial_{t} \mathbf{v}+\operatorname{div}_{x}(\mathbf{v} \otimes \mathbf{v})-\operatorname{div}_{x} \mathbb{S}+\nabla_{x} p-\varepsilon\left(\Delta_{x} \mathbf{v}+\operatorname{div}_{x}\left(\left|\mathbb{D}_{x} \mathbf{v}\right|^{\frac{1}{5}} \mathbb{D}_{x} \mathbf{v}\right)\right) & =\mathbf{b} \\
\operatorname{div}_{x} \mathbf{v} & =0
\end{aligned}
$$

with

$$
\mathbb{S}=\left(\kappa+\left|\mathbb{D}_{x} \mathbf{v}\right|^{2}\right)^{\frac{q-2}{2}} \mathbb{D}_{x} \mathbf{v}
$$


and $(t, x) \in(0, T) \times \Omega$ and boundary condition

$$
\mathbf{v}=0 \text { on }(0, T) \times \partial \Omega .
$$

In this section we first assume $\kappa>0$, subsequently we let $\kappa \rightarrow 0$. It is worth remarking that being $q \in\left(\frac{6}{5}, \frac{11}{5}\right)$, we need to approximate the degenerate stress tensor with a non-degenerate one in order to ensure the uniqueness of the Galerkin approximation, which is crucial for our argument. Moreover once we added the $p$-Laplacian term with $p=11 / 5$ we may conclude the existence of time-periodic Galerkin approximations to the approximating system (2.43) by virtue of the results proved in the former section; however for the sake of clarity and for the reader's convenience we perform the main steps below.

Given $\mathbf{v}_{0}^{n} \in \operatorname{span}\left\{\boldsymbol{\omega}^{1}, \ldots, \boldsymbol{\omega}^{n}\right\}$ such that

$$
\left\|\mathbf{v}_{0}^{n}\right\|_{L^{2}\left(\Omega ; R^{3}\right)} \leq K:=\left(\frac{C_{2}\left(\max _{[0, T]}\|\mathbf{b}\|_{L^{2}\left(\Omega_{T} ; R^{3}\right)}^{q^{\prime}}+\kappa^{\frac{q}{2}}\right)}{C_{1} C_{S}}\right)^{\frac{1}{q}}
$$

with constants $C_{1}, C_{2}, C_{S}$ defined later in the proof, we look for the Galerkin approximation

$$
\mathbf{v}^{n}(t, x):=\sum_{i=1}^{n} c_{i}^{n}(t) \boldsymbol{\omega}^{i}(x)
$$

such that it satisfies for any $k=1, \ldots, n$

$$
\begin{aligned}
& \left(\partial_{t} \mathbf{v}^{n}, \vec{\omega}^{k}\right)+\left(\mathbb{S}^{n}-\mathbf{v}^{n} \otimes \mathbf{v}^{n}, \mathbb{D}_{x} \boldsymbol{\omega}^{k}\right)+\varepsilon\left(\nabla_{x} \mathbf{v}^{n}, \nabla_{x} \boldsymbol{\omega}^{k}\right) \\
& \quad+\varepsilon\left(\left|\mathbb{D}_{x} \mathbf{v}^{n}\right|^{\frac{1}{5}} \mathbb{D}_{x} \mathbf{v}^{n}, \mathbb{D}_{x} \boldsymbol{\omega}^{k}\right)=\left(\mathbf{b}, \boldsymbol{\omega}^{k}\right) \\
& \mathbf{v}^{n}(0)=\mathbf{v}_{0}^{n}
\end{aligned}
$$

where

$$
\mathbb{S}^{n}:=\left(\kappa+\left|\mathbb{D}_{x} \mathbf{v}^{n}\right|^{2}\right)^{\frac{q-2}{2}} \mathbb{D}_{x} \mathbf{v}^{n},
$$

meaning in components that for any $k=1, \ldots, n$

$$
\begin{aligned}
\left(c_{k}^{n}(t)\right)^{\prime}= & \sum_{i, j=1}^{n} c_{i}^{n} c_{j}^{n} f_{i j k}-\left(\mathbb{S}^{n}, \mathbb{D}_{x} \boldsymbol{\omega}_{k}\right)-\varepsilon \sum_{i=1}^{n} c_{i}^{n} g_{i k} \\
& -\varepsilon\left(\left|\mathbb{D}_{x} \mathbf{v}^{n}\right|^{\frac{1}{5}} \mathbb{D}_{x} \mathbf{v}^{n}, \mathbb{D}_{x} \boldsymbol{\omega}^{k}\right)+b_{k}, \\
c_{k}^{n}(0)= & \left(\mathbf{v}_{0}^{n}, \boldsymbol{\omega}^{k}\right),
\end{aligned}
$$

and where we introduced the notation

$$
f_{i j k}:=\left(\boldsymbol{\omega}^{i} \otimes \boldsymbol{\omega}^{j}, \nabla_{x} \boldsymbol{\omega}^{k}\right), g_{i k}:=\left(\nabla_{x} \boldsymbol{\omega}^{i}, \nabla_{x} \boldsymbol{\omega}^{k}\right), b_{k}:=\left(\mathbf{b}, \boldsymbol{\omega}^{k}\right) .
$$

The existence of a solution $\mathbf{c}^{n}(t):=\left(c_{1}^{n}, \ldots, c_{n}^{n}\right)$ to the Cauchy problem (2.46) in a local time interval $\left[0, t_{n}\right)$ follows by standard results on ODEs. Thanks to the presence of $\kappa>0$, we can state that the right-hand side of (2.46a) is a Lipschitz function and thus the uniqueness of $\mathbf{c}^{n}(t)$ follows. Now, let us multiply (2.45a) by $c_{k}^{n}$ and sum over $k=1, \ldots, n$

$$
\begin{aligned}
& \frac{\mathrm{d}}{\mathrm{d} t}\left\|\mathbf{v}^{n}(t)\right\|_{L^{2}\left(\Omega ; R^{3}\right)}^{2}+C_{1}\left\|\mathbb{D}_{x} \mathbf{v}^{n}\right\|_{L^{q}\left(\Omega ; R^{3 \times 3}\right)}^{q}+\varepsilon\left\|\nabla_{x} \mathbf{v}^{n}\right\|_{L^{2}\left(\Omega ; R^{3 \times 3}\right)}^{2} \\
& \quad+\varepsilon\left\|\mathbb{D}_{x} \mathbf{v}^{n}\right\|_{L^{\frac{11}{5}}\left(\Omega ; R^{3 \times 3}\right)}^{\frac{11}{5}} \leq C_{2}\|\mathbf{b}\|_{L^{2}\left(\Omega ; R^{3}\right)}^{q^{\prime}}+C_{2} \kappa^{\frac{q}{2}},
\end{aligned}
$$

then let us integrate in time over $(0, t)$, we obtain for all $t \in[0, T]$

$$
\left\|\mathbf{v}^{n}(t)\right\|_{L^{2}\left(\Omega ; R^{3}\right)}^{2} \leq\left\|\mathbf{v}_{0}^{n}\right\|_{L^{2}\left(\Omega ; R^{3}\right)}^{2}+C_{2} T \max _{[0, T]}\|\mathbf{b}\|_{L^{2}\left(\Omega ; R^{3}\right)}^{q^{\prime}}+C_{2} \kappa^{\frac{q}{2}} T
$$

where $\left\|\mathbf{v}^{n}(t)\right\|_{L^{2}\left(\Omega ; R^{3}\right)}^{2}=\left|\mathbf{c}^{n}(t)\right|^{2}$. This implies that $\mathbf{v}^{n}(t)$ is well-defined on the whole interval $[0, T]$ and $\mathbf{v}^{n}(t) \in C\left([0, T] ; L^{2}\left(\Omega ; R^{3}\right)\right)$. 
Let us introduce the mapping

$$
F: \mathbb{R}^{n} \rightarrow \mathbb{R}^{n}, F\left(\mathbf{c}_{0}^{n}\right):=\mathbf{c}^{n}(T)
$$

where $\mathbf{c}^{n}(t)$ is the unique solution to the system (2.46) corresponding to the initial value $\mathbf{c}_{0}^{n}$. Repeating the arguments showed in the analogous paragraph in the case $q \geq 11 / 5$, we obtain that $F$ transforms the ball $B_{K}$ of $R^{n}$ of radius $K$ into itself and it is continuous. Therefore by the Brouwer theorem it follows the existence of a fixed point, which means that for any $n \in N$ there exists $\mathbf{v}^{n}(t)$ solution to (2.45a) such that $\mathbf{v}^{n}(T)=\mathbf{v}^{n}(0)$.

Limit as $n \rightarrow+\infty$. Integrating (2.47) in time over $(0, T)$ we have

$$
\begin{aligned}
& \sup _{n \in N}\left(\max _{t \in[0, T]}\left\|\mathbf{v}^{n}(t)\right\|_{L^{2}\left(\Omega ; R^{3}\right)}+\left\|\mathbb{D}_{x} \mathbf{v}^{n}\right\|_{L^{q}\left(\Omega_{T} ; R^{3 \times 3}\right)}\right. \\
& \left.\quad+\left\|\varepsilon^{\frac{1}{2}} \nabla_{x} \mathbf{v}^{n}\right\|_{L^{2}\left(\Omega_{T} ; R^{3 \times 3}\right)}+\left\|\varepsilon^{\frac{5}{11}} \mathbb{D}_{x} \mathbf{v}^{n}\right\|_{L^{\frac{11}{5}}\left(\Omega_{T} ; R^{3 \times 3}\right)}\right)<+\infty,
\end{aligned}
$$

and as a consequence

$$
\sup _{n \in N}\left\|\mathbb{S}^{n}\right\|_{L^{q^{\prime}}\left(\Omega_{T} ; R^{3 \times 3}\right)}<+\infty .
$$

Analogously to (2.18), it holds

$$
\int_{0}^{T} \int_{\Omega}\left|\mathbf{v}^{n}\right|^{\frac{11}{3}} \mathrm{~d} x \mathrm{~d} t \leq \sup _{[0, T]}\left(\int_{\Omega}\left|\mathbf{v}^{n}\right|^{2} \mathrm{~d} x\right)^{\frac{11}{15}} \int_{0}^{T} \int_{\Omega}\left|\nabla_{x} \mathbf{v}^{n}\right|^{\frac{11}{5}} \mathrm{~d} x \mathrm{~d} t,
$$

then the Korn inequality, and (2.49) imply that

$$
\sup _{n \in N}\left\|\mathbf{v}^{n}\right\|_{L^{\frac{11}{3}}\left(\Omega_{T} ; R^{3}\right)}<+\infty .
$$

By virtue of estimates (2.48)-(2.51) we derive that the time derivative of $\mathbf{v}^{n}$ is uniformly bounded in the following space:

$$
\sup _{n \in N}\left\|\partial_{t} \mathbf{v}^{n}\right\|_{(L}^{\left.\frac{11}{5}\left(0, T ; W_{0, \text { div }}^{1, \frac{11}{5}}\left(\Omega ; R^{3}\right)\right)\right)^{*}}<+\infty
$$

The obtained uniform estimates yield the existence of $\mathbf{v}$ and $\mathbb{S}$ such that, for suitable subsequence not relabelled, the following convergences hold:

$$
\begin{aligned}
& \mathbf{v}^{n} \rightarrow \mathbf{v} \text { weakly-* in } L^{\infty}\left(0, T ; L^{2}\left(\Omega ; R^{3}\right)\right), \\
& \mathbf{v}^{n} \rightarrow \mathbf{v} \text { weakly in } L^{\frac{11}{5}}\left(0, T ; W_{0, \operatorname{div}}^{1, \frac{11}{5}}\left(\Omega ; R^{3}\right)\right), \\
& \partial_{t} \mathbf{v}^{n} \rightarrow \partial_{t} \mathbf{v} \text { weakly in }\left(L^{\frac{11}{5}}\left(0, T ; W_{0, \frac{11}{5}}^{1,}\left(\Omega ; R^{3}\right)\right)\right)^{*}, \\
& \mathbb{S}^{n} \rightarrow \mathbb{S} \text { weakly in } L^{q^{\prime}}\left(\Omega_{T} ; R^{3 \times 3}\right)
\end{aligned}
$$

The Aubin-Lions compactness lemma applied to $W_{0, \operatorname{div}}^{1, \frac{11}{5}} \hookrightarrow \hookrightarrow L^{\frac{11}{5}} \hookrightarrow\left(W_{0, \text { div }}^{1, \frac{11}{5}}\right)^{*}$ and the interpolation inequality in (2.26) with $q=11 / 5$ imply that

$$
\mathbf{v}^{n} \rightarrow \mathbf{v} \text { strongly in } L^{r}\left(\Omega_{T} ; R^{3}\right) \text { for any } r<\frac{11}{3} .
$$

On the other side, from (2.44) it follows that for a suitable subsequence it holds

$$
\mathbf{v}_{0}^{n} \rightarrow \mathbf{v}_{0} \text { weakly in } L^{2}\left(\Omega ; R^{3}\right) \text { and }\left\|\mathbf{v}_{0}\right\|_{L^{2}\left(\Omega ; R^{3}\right)} \leq K .
$$

Now, we can follow step by step the passage to the limit as $n \rightarrow+\infty$ in the section $q \geq 11 / 5$, and we obtain $\mathbf{v}$ time-periodic weak solution to system (2.43) with period $T$.

Limit as $\kappa \rightarrow 0$. Therefore for any $\kappa>0$ we proved the existence of a solution $\mathbf{v}^{\kappa}$ time-periodic with period $T$ corresponding to initial data $\mathbf{v}_{0}^{\kappa}$ whose $L^{2}$-norm is bounded by the constant $K$ given in (2.44). 
Since we may assume $\kappa<1,\left\|\mathbf{v}_{0}^{\kappa}\right\|_{L^{2}\left(\Omega ; R^{3}\right)}$ is uniformly bounded in $\kappa$, hence there exists a subsequence, still denoted by $\mathbf{v}_{0}^{\kappa}$, weakly converging in $L^{2}\left(\Omega ; R^{3}\right)$ to a limit $\mathbf{v}_{0}$ such that

$$
\left\|\mathbf{v}_{0}\right\|_{L^{2}\left(\Omega ; R^{3}\right)} \leq \bar{K}:=\left(\frac{C_{2}\left(\max _{[0, T]}\|\mathbf{b}\|_{L^{2}\left(\Omega_{T} ; R^{3}\right)}^{q^{\prime}}+1\right)}{C_{1} C_{S}}\right)^{\frac{1}{q}} .
$$

Next, the sequence $\left\{\mathbf{v}^{\kappa}\right\}$ satisfies the bounds in (2.49) uniformly in $\kappa$. Since

$$
\begin{aligned}
\int_{0}^{T} & \int_{\Omega}\left(\left(\kappa+\left|\mathbb{D}_{x} \mathbf{v}\right|^{2}\right)^{\frac{q-2}{2}}\left|\mathbb{D}_{x} \mathbf{v}\right|\right)^{q^{\prime}} \mathrm{d} x \mathrm{~d} t \\
\quad \leq & \int_{0}^{T} \int_{\Omega}\left(\kappa+\left|\mathbb{D}_{x} \mathbf{v}\right|^{2}\right)^{\frac{(q-2) q}{2(q-1)}}\left(\kappa+\left|\mathbb{D}_{x} \mathbf{v}\right|^{2}\right)^{\frac{q}{2(q-1)}} \mathrm{d} x \mathrm{~d} t \\
\quad & \int_{0}^{T} \int_{\Omega}\left(\kappa+\left|\mathbb{D}_{x} \mathbf{v}\right|^{2}\right)^{\frac{q}{2}} \mathrm{~d} x \mathrm{~d} t \\
& \leq \int_{\left|\mathbb{D}_{x} \mathbf{v}\right|^{2} \geq \kappa}\left(2\left|\mathbb{D}_{x} \mathbf{v}\right|^{2}\right)^{\frac{q}{2}} \mathrm{~d} x \mathrm{~d} t+\int_{\left|\mathbb{D}_{x} \mathbf{v}\right|^{2} \leq \kappa}(2 \kappa)^{\frac{q}{2}} \mathrm{~d} x \mathrm{~d} t
\end{aligned}
$$

we can state that $\mathbb{S}^{\kappa}:=\left(\kappa+\left|\mathbb{D}_{x} \mathbf{v}^{\kappa}\right|^{2}\right)^{\frac{q-2}{2}} \mathbb{D}_{x} \mathbf{v}^{\kappa}$ is uniformly bounded in $L^{q^{\prime}}\left(\Omega_{T} ; R^{3 \times 3}\right)$, moreover $\mathbf{v}^{\kappa}$ enjoys also the bounds in $(2.51)$ and the sequence $\left\{\partial_{t} \mathbf{v}^{\kappa}\right\}$ is bounded in $\left(L^{\frac{11}{5}}\left(0, T ; W_{0}^{1, \frac{11}{5}}\left(\Omega ; R^{3}\right)\right)\right)^{*}$ uniformly in $\kappa$. Hence, employing also the Aubin-Lions compactness lemma, there exist subsequences $\mathbf{v}^{\kappa}, \mathbb{S}^{\kappa}$, not relabelled, weakly, weakly-* and strongly converging in the relevant spaces to the limit $\mathbf{v}$ and $\mathbb{S}$ respectively. We may follow the steps of the passage to the limit in the previous paragraph and obtain that

$$
\begin{aligned}
& \int_{0}^{T}\left\langle\partial_{t} \mathbf{v}, \boldsymbol{\varphi}\right\rangle \mathrm{d} t+\int_{0}^{T} \int_{\Omega} \mathbb{S}: \mathbb{D}_{x} \boldsymbol{\varphi} \mathrm{d} x \mathrm{~d} t-\int_{0}^{T} \int_{\Omega} \mathbf{v} \otimes \mathbf{v}: \mathbb{D}_{x} \boldsymbol{\varphi} \mathrm{d} x \mathrm{~d} t \\
& \quad+\varepsilon \int_{0}^{T} \int_{\Omega} \nabla_{x} \mathbf{v}: \nabla_{x} \boldsymbol{\varphi} \mathrm{d} x \mathrm{~d} t+\varepsilon \int_{0}^{T} \int_{\Omega}\left|\mathbb{D}_{x} \mathbf{v}\right|^{\frac{1}{5}} \mathbb{D}_{x} \mathbf{v}: \mathbb{D}_{x} \boldsymbol{\varphi} \mathrm{d} x \mathrm{~d} t \\
& =\int_{0}^{T} \int_{\Omega} \mathbf{b} \cdot \boldsymbol{\varphi} \mathrm{d} x \mathrm{~d} t \text { for any } \boldsymbol{\varphi} \in L^{\frac{11}{5}}\left(0, T ; W_{0, \text { div }}^{1, \frac{11}{5}}\left(\Omega ; R^{3}\right)\right) .
\end{aligned}
$$

It remains to be proved the identification of the limit $\mathbb{S}$. Since

$$
\left.\left.\left|\left(\kappa+\left|\mathbb{D}_{x} \varphi\right|^{2}\right)^{\frac{q-2}{2}} \mathbb{D}_{x} \boldsymbol{\varphi}-\right| \mathbb{D}_{x} \varphi\right|^{q-2} \mathbb{D}_{x} \varphi\right|^{q^{\prime}} \rightarrow 0 \text { as } \kappa \rightarrow 0 \text {, a.e. in } \Omega_{T},
$$

and

$$
\left.\left.\left|\left(\kappa+\left|\mathbb{D}_{x} \varphi\right|^{2}\right)^{\frac{q-2}{2}} \mathbb{D}_{x} \varphi-\right| \mathbb{D}_{x} \varphi\right|^{q-2} \mathbb{D}_{x} \varphi\right|^{q^{\prime}} \leq(C+2)\left|\mathbb{D}_{x} \varphi\right|^{(q-2) q^{\prime}}\left|\mathbb{D}_{x} \varphi\right|^{q^{\prime}} \leq C\left|\mathbb{D}_{x} \varphi\right|^{q}
$$

where $C$ is a constant independent of $\kappa$ and varying from line to line, the Lebesgue dominated convergence theorem ensures that

$$
\left(\kappa+\left|\mathbb{D}_{x} \varphi\right|^{2}\right)^{\frac{q-2}{2}} \mathbb{D}_{x} \varphi \rightarrow\left|\mathbb{D}_{x} \varphi\right|^{q-2} \mathbb{D}_{x} \varphi \text { strongly in } L^{q^{\prime}}\left(\Omega_{T} ; R^{3 \times 3}\right) .
$$

The monotonicity of the operator (1.1c) implies that the quantity

$$
\int_{0}^{T} \int_{\Omega}\left[\left(\kappa+\left|\mathbb{D}_{x} \mathbf{v}^{\kappa}\right|^{2}\right)^{\frac{q-2}{2}} \mathbb{D}_{x} \mathbf{v}^{\kappa}-\left(\kappa+\left|\mathbb{D}_{x} \boldsymbol{\varphi}\right|^{2}\right)^{\frac{q-2}{2}} \mathbb{D}_{x} \boldsymbol{\varphi}\right]:\left(\mathbb{D}_{x} \mathbf{v}^{\kappa}-\mathbb{D}_{x} \boldsymbol{\varphi}\right) \mathrm{d} x \mathrm{~d} t
$$

is non-negative and, using $(2.45 \mathrm{a})$, it can be rewritten as

$$
\begin{aligned}
& \int_{0}^{T} \int_{\Omega}\left[\mathbf{b} \cdot \mathbf{v}^{\kappa}-\varepsilon\left|\nabla_{x} \mathbf{v}^{\kappa}\right|^{2}-\varepsilon\left|\mathbb{D}_{x} \mathbf{v}^{\kappa}\right|^{\frac{11}{5}}-\left(\kappa+\left|\mathbb{D}_{x} \mathbf{v}^{\kappa}\right|^{2}\right)^{\frac{q-2}{2}} \mathbb{D}_{x} \mathbf{v}^{\kappa}: \mathbb{D}_{x} \boldsymbol{\varphi}\right. \\
& \left.\quad-\left(\kappa+\left|\mathbb{D}_{x} \boldsymbol{\varphi}\right|^{2}\right)^{\frac{q-2}{2}} \mathbb{D}_{x} \boldsymbol{\varphi}:\left(\mathbb{D}_{x} \mathbf{v}^{\kappa}-\mathbb{D}_{x} \boldsymbol{\varphi}\right)\right] \mathrm{d} x \mathrm{~d} t \geq 0 .
\end{aligned}
$$


Employing the strong convergence (2.61), the boundedness of $\left\|\mathbb{D}_{x} \mathbf{v}^{\kappa}\right\|_{L^{q}\left(\Omega_{T} ; R^{3 \times 3}\right)}$ uniformly in $\kappa$, and the weak convergence of $\mathbb{D}_{x} \mathbf{v}^{\kappa}$ to $\mathbb{D}_{x} \mathbf{v}$ in $L^{q}\left(\Omega_{T} ; R^{3 \times 3}\right)$, it follows that

$$
\begin{aligned}
\int_{0}^{T} & \int_{\Omega}\left(\kappa+\left|\mathbb{D}_{x} \boldsymbol{\varphi}\right|^{2}\right)^{\frac{q-2}{2}} \mathbb{D}_{x} \boldsymbol{\varphi}:\left(\mathbb{D}_{x} \mathbf{v}^{\kappa}-\mathbb{D}_{x} \boldsymbol{\varphi}\right) \mathrm{d} x \mathrm{~d} t \\
= & \int_{0}^{T} \int_{\Omega}\left(\left(\kappa+\left|\mathbb{D}_{x} \boldsymbol{\varphi}\right|^{2}\right)^{\frac{q-2}{2}} \mathbb{D}_{x} \boldsymbol{\varphi}-\left|\mathbb{D}_{x} \boldsymbol{\varphi}\right|^{q-2} \mathbb{D}_{x} \boldsymbol{\varphi}\right): \mathbb{D}_{x} \mathbf{v}^{\kappa} \mathrm{d} x \mathrm{~d} t \\
& +\int_{0}^{T} \int_{\Omega}\left|\mathbb{D}_{x} \boldsymbol{\varphi}\right|^{q-2} \mathbb{D}_{x} \boldsymbol{\varphi}: \mathbb{D}_{x} \mathbf{v}^{\kappa} \mathrm{d} x \mathrm{~d} t \\
& -\int_{0}^{T} \int_{\Omega}\left(\kappa+\left|\mathbb{D}_{x} \varphi\right|^{2}\right)^{\frac{q-2}{2}} \mathbb{D}_{x} \boldsymbol{\varphi}: \mathbb{D}_{x} \boldsymbol{\varphi} \mathrm{d} x \mathrm{~d} t \\
& \longrightarrow \int_{0}^{T} \int_{\Omega}\left|\mathbb{D}_{x} \boldsymbol{\varphi}\right|^{q-2} \mathbb{D}_{x} \boldsymbol{\varphi}:\left(\mathbb{D}_{x} \mathbf{v}-\mathbb{D}_{x} \boldsymbol{\varphi}\right) \mathrm{d} x \mathrm{~d} t
\end{aligned}
$$

Then taking the liminf in (2.62) and using all the collected convergences we get

$$
\begin{aligned}
0 & \leq \int_{0}^{T} \int_{\Omega}\left[\mathbf{b} \cdot \mathbf{v}-\varepsilon\left|\nabla_{x} \mathbf{v}\right|^{2}-\varepsilon\left|\mathbb{D}_{x} \mathbf{v}\right|^{\frac{11}{5}}\right. \\
& \left.-\mathbb{S}: \mathbb{D}_{x} \boldsymbol{\varphi}-\left|\mathbb{D}_{x} \boldsymbol{\varphi}\right|^{q-2} \mathbb{D}_{x} \boldsymbol{\varphi}:\left(\mathbb{D}_{x} \mathbf{v}-\mathbb{D}_{x} \boldsymbol{\varphi}\right)\right] \mathrm{d} x \mathrm{~d} t .
\end{aligned}
$$

At this level of approximation, due to the presence of $\varepsilon>0$, we can still use the weak solution itself $\mathbf{v}$ as test function in (2.60) and comparing the outcome with (2.64), it follows that

$$
0 \leq \int_{0}^{T} \int_{\Omega}\left(\mathbb{S}-\left|\mathbb{D}_{x} \varphi\right|^{q-2} \mathbb{D}_{x} \boldsymbol{\varphi}\right):\left(\mathbb{D}_{x} \mathbf{v}-\mathbb{D}_{x} \boldsymbol{\varphi}\right) \mathrm{d} x \mathrm{~d} t .
$$

Now, choosing $\varphi:=\mathbf{v} \pm \lambda \mathbf{w}$ with $\lambda>0$, dividing by $\lambda$ and then taking the limit as $\lambda \rightarrow 0$ we obtain that

$$
\mathbb{S}=\left|\mathbb{D}_{x} \mathbf{v}\right|^{q-2} \mathbb{D}_{x} \mathbf{v} \text { a.e. in } \Omega_{T} .
$$

Limit as $\varepsilon \rightarrow 0$. We constructed a sequence $\left\{\mathbf{v}^{\varepsilon}\right\}$ of time-periodic solution with period $T$ to (2.60) with viscous stress tensor $\left\{\mathbb{S}^{\varepsilon}\right\}$ fulfilling (2.66), corresponding to a sequence of initial data $\left\{\mathbf{v}_{0}^{\varepsilon}\right\}$ satisfying (2.59). Taking as test function $\varphi=\mathbf{v}^{\varepsilon}$ in (2.60) we derive the following uniform estimates

$$
\begin{aligned}
& \sup _{\varepsilon>0}\left(\max _{t \in[0, T]}\left\|\mathbf{v}^{\epsilon}(t)\right\|_{L^{2}(\Omega)}+\left\|\mathbb{D}_{x} \mathbf{v}^{\varepsilon}\right\|_{L^{q}\left(\Omega_{T} ; R^{3 \times 3}\right)}\right. \\
& \left.\quad+\left\|\varepsilon^{\frac{1}{2}} \nabla \mathbf{v}^{\varepsilon}\right\|_{L^{2}\left(\Omega_{T} ; R^{3 \times 3}\right)}+\left\|\varepsilon^{\frac{5}{11}} \mathbb{D}_{x} \mathbf{v}^{\varepsilon}\right\|_{\left.L^{\frac{11}{5}}\left(\Omega_{T} ; R^{3 \times 3}\right)\right)}\right)<+\infty .
\end{aligned}
$$

As a consequence and similarly to the section $q \geq 11 / 5$ we get

$$
\sup _{\varepsilon>0}\left(\left\|\mathbb{S}^{\varepsilon}\right\|_{L^{q^{\prime}}\left(\Omega_{T} ; R^{3 \times 3}\right)}+\left\|\mathbf{v}^{\varepsilon}\right\|_{L^{\frac{5 q}{3}}\left(\Omega_{T} ; R^{3}\right)}\right)<+\infty .
$$

Then, it follows

$$
\sup _{\varepsilon>0}\left\|\partial_{t} \mathbf{v}^{\varepsilon}\right\|_{\left(L^{\frac{5 q}{5 q-6}}\left(0, T ; W_{0, \operatorname{div}}^{1, \frac{5 q}{5 q-6}}\left(\Omega ; R^{3}\right)\right)\right)^{*}}<+\infty
$$

where we used that when $q \in(6 / 5,11 / 5)$ the space $L^{q}\left(0, T ; W_{0}^{1, q}\left(\Omega ; R^{3}\right)\right) \cap L^{\frac{5 q}{5 q-6}}\left(0, T ; W_{0}^{1, \frac{5 q}{5 q-6}}\left(\Omega ; R^{3}\right)\right)$ coincides with $L^{\frac{5 q}{5 q-6}}\left(0, T ; W_{0}^{1, \frac{5 q}{5 q-6}}\left(\Omega ; R^{3}\right)\right)$. 
Repeating the same arguments perfomed in the section $q \geq 11 / 5$, we obtain the existence of $\mathbf{v}$ and $\mathbb{S}$ such that, for suitable subsequences not relabelled, the following convergences hold:

$$
\begin{array}{rlrl}
\mathbf{v}^{\varepsilon} & \rightarrow \mathbf{v} & \text { weakly-* in } L^{\infty}\left(0, T ; L^{2}\left(\Omega ; R^{3}\right)\right), \\
\mathbf{v}^{\varepsilon} & \rightarrow \mathbf{v} & \text { weakly in } L^{q}\left(0, T ; W_{0, \text { div }}^{1, q}\left(\Omega ; R^{3}\right)\right), \\
\partial_{t} \mathbf{v}^{\varepsilon} \rightarrow \partial_{t} \mathbf{v} & \text { weakly in }\left(L^{\frac{5 q}{5 q-6}}\left(0, T ; W_{0, \text { div }}^{1, \frac{5 q}{5 q-6}}\left(\Omega ; R^{3}\right)\right)\right)^{*}, \\
\mathbf{v}^{\varepsilon} \rightarrow \mathbf{v} & \text { strongly in } L^{r}\left(\Omega_{T} ; R^{3}\right) \text { for any } r<\frac{5 q}{3}, \\
\mathbb{S}^{\varepsilon} \rightarrow \overline{\mathbb{S}} & \text { weakly in } L^{q^{\prime}}\left(\Omega_{T} ; R^{3 \times 3}\right) .
\end{array}
$$

Furthermore,

$$
\begin{aligned}
\left|\varepsilon \int_{0}^{T} \int_{\Omega} \nabla_{x} \mathbf{v}^{\varepsilon}: \nabla_{x} \boldsymbol{\varphi} \mathrm{d} x \mathrm{~d} t\right| & \leq \varepsilon\left\|\nabla_{x} \mathbf{v}^{\varepsilon}\right\|_{\frac{5 q}{6}}\left\|\nabla_{x} \boldsymbol{\varphi}\right\|_{\frac{5 q}{5 q-6}} \\
& \leq \varepsilon C\left\|\nabla_{x} \boldsymbol{\varphi}\right\|_{\frac{5 q}{5 q-6}} \rightarrow 0 \text { as } \varepsilon \rightarrow 0
\end{aligned}
$$

and

$$
\begin{aligned}
& \left.\left|\varepsilon \int_{0}^{T} \int_{\Omega}\right| \mathbb{D}_{x} \mathbf{v}^{\varepsilon}\right|^{\frac{1}{5}} \mathbb{D}_{x} \mathbf{v}^{\varepsilon}:\left.\mathbb{D}_{x} \boldsymbol{\varphi} \mathrm{d} x \mathrm{~d} t\left|\leq \varepsilon^{\frac{5}{11}} \int_{0}^{T} \int_{\Omega} \varepsilon^{\frac{6}{11}}\right| \mathbb{D}_{x} \mathbf{v}^{\varepsilon}\right|^{\frac{6}{5}}\left|\mathbb{D}_{x} \boldsymbol{\varphi}\right| \mathrm{d} x \mathrm{~d} t \\
& \quad \leq \varepsilon^{\frac{5}{11}}\left(\int_{0}^{T} \int_{\Omega} \varepsilon\left|\mathbb{D}_{x} \mathbf{v}^{\varepsilon}\right|^{\frac{11}{5}} \mathrm{~d} x \mathrm{~d} t\right)^{\frac{6}{11}}\left\|\mathbb{D}_{x} \boldsymbol{\varphi}\right\|_{L^{\frac{11}{5}}}\left(\Omega_{T} ; R^{3 \times 3}\right) \\
& =\varepsilon^{\frac{5}{11}}\left\|\varepsilon^{\frac{5}{11}} \mathbb{D}_{x} \mathbf{v}^{\varepsilon}\right\|_{\frac{11}{5}}^{\frac{6}{5}}\left\|\mathbb{D}_{x} \boldsymbol{\varphi}\right\|_{\frac{11}{5}} \leq \varepsilon^{\frac{5}{11}} C\left\|\mathbb{D}_{x} \boldsymbol{\varphi}\right\|_{\frac{11}{5}} \rightarrow 0 \text { as } \varepsilon \rightarrow 0 .
\end{aligned}
$$

Then taking the limit as $\varepsilon \rightarrow 0$ in $(2.60)$ and by a standard density argument we obtain that $\mathbf{v}$ and $\overline{\mathbb{S}}$ fulfil the following weak formulation:

$$
\begin{gathered}
\int_{0}^{T}\left\langle\partial_{t} \mathbf{v}, \boldsymbol{\varphi}\right\rangle \mathrm{d} t-\int_{0}^{T} \int_{\Omega} \mathbf{v} \otimes \mathbf{v}: \nabla_{x} \boldsymbol{\varphi} \mathrm{d} x \mathrm{~d} t+\int_{0}^{T} \int_{\Omega} \overline{\mathbb{S}}: \mathbb{D}_{x} \boldsymbol{\varphi} \mathrm{d} x \mathrm{~d} t \\
=\int_{0}^{T} \int_{\Omega} \mathbf{b} \cdot \boldsymbol{\varphi} \mathrm{d} x \mathrm{~d} t \text { for any } \boldsymbol{\varphi} \in C^{\infty}\left((0, T) ; C_{0, \text { div }}^{\infty}\left(\Omega ; R^{3}\right)\right) .
\end{gathered}
$$

From the identity $(2.77)$ it is standard to establish $\mathbf{v} \in C_{\text {weak }}\left([0, T] ; L^{2}\left(\Omega ; R^{3}\right)\right)$ see e.g. [6, Step 4, Proof of Theorem 3.3].

Thanks to (2.67) the following convergence, for a suitable subsequence not relabelled, holds:

$$
\left(\mathbf{v}^{\varepsilon}(T), \varphi\right) \rightarrow(\mathbf{v}(T), \varphi) \text { as } \varepsilon \rightarrow 0
$$

for any $\varphi \in C_{0}^{\infty}\left(\Omega ; R^{3}\right)$. On the other hand, by $(2.59)$ we get that

$$
\mathbf{v}_{0}^{\varepsilon} \rightarrow \mathbf{v}_{0} \text { weakly in } L^{2}\left(\Omega ; R^{3}\right)
$$

for a subsequence not relabelled. Therefore it holds

$$
\left(\mathbf{v}(T)-\mathbf{v}_{0}, \boldsymbol{\varphi}\right)=\lim _{\varepsilon \rightarrow 0}\left(\mathbf{v}^{\varepsilon}(T)-\mathbf{v}_{0}^{\varepsilon}, \boldsymbol{\varphi}\right)=0 \quad \forall \boldsymbol{\varphi} \in C_{0}^{\infty}\left(\Omega ; R^{3}\right),
$$


which means that the limit function $\mathbf{v}$ is time-periodic with period $T$. It remains to identify the rheological law. We perform the Lipschitz truncation method in the solenoidal version. By (2.77), from the weak formulation fulfilled by the approximation $\mathbf{v}^{\varepsilon}$ i.e. (2.60), and taking into account the time-periodicity of $\mathbf{v}^{\varepsilon}$ and $\mathbf{v}$, it is easily seen that

$$
\begin{aligned}
& -\int_{0}^{T} \int_{\Omega}\left(\mathbf{v}^{\varepsilon}-\mathbf{v}\right) \cdot \partial_{t} \boldsymbol{\varphi} \mathrm{d} x \mathrm{~d} t+\int_{0}^{T} \int_{\Omega}\left(\mathbb{S}^{\varepsilon}-\overline{\mathbb{S}}\right): \mathbb{D}_{x} \boldsymbol{\varphi} \mathrm{d} x \mathrm{~d} t \\
& \quad+\varepsilon \int_{0}^{T} \int_{\Omega} \nabla_{x} \mathbf{v}^{\varepsilon}: \nabla_{x} \boldsymbol{\varphi} \mathrm{d} x \mathrm{~d} t+\varepsilon \int_{0}^{T} \int_{\Omega}\left|\mathbb{D}_{x} \mathbf{v}^{\varepsilon}\right|^{\frac{1}{5}} \mathbb{D}_{x} \mathbf{v}^{\varepsilon}: \mathbb{D}_{x} \boldsymbol{\varphi} \mathrm{d} x \mathrm{~d} t \\
& \quad=\int_{0}^{T} \int_{\Omega}\left(\mathbf{v}^{\varepsilon} \otimes \mathbf{v}^{\varepsilon}-\mathbf{v} \otimes \mathbf{v}\right): \nabla_{x} \boldsymbol{\varphi} \mathrm{d} x \mathrm{~d} t
\end{aligned}
$$

for any $\varphi \in C^{\infty}\left((0, T) ; C_{0, \text { div }}^{\infty}\left(\Omega ; R^{3}\right)\right)$. Let us set $\mathbf{u}^{\varepsilon}:=\mathbf{v}^{\varepsilon}-\mathbf{v}$ and $\mathbb{H}_{1}^{\varepsilon}:=\mathbb{S}^{\varepsilon}-\overline{\mathbb{S}}$, then let us rewrite convergences $(2.70)-(2.74)$ as follows:

$$
\begin{array}{ll}
\mathbf{u}^{\varepsilon} \rightarrow 0 & \text { weakly-* in } L^{\infty}\left(0, T ; L^{2}\left(\Omega ; R^{3}\right)\right), \\
\mathbf{u}^{\varepsilon} \rightarrow 0 & \text { weakly in } L^{q}\left(0, T ; W_{0, \text { div }}^{1, q}\left(\Omega ; R^{3}\right)\right), \\
\mathbf{u}^{\varepsilon} \rightarrow 0 & \text { strongly in } \left.L^{r}\left(\Omega_{T} ; R^{3}\right)\right) \text { for any } 1<r<\frac{5 q}{3}, \\
\mathbb{H}_{1}^{\varepsilon} \rightarrow 0 & \text { weakly in } L^{q^{\prime}}\left(\Omega_{T} ; R^{3 \times 3}\right) .
\end{array}
$$

Further, since for any $1<r<5 q / 6$ it holds

$$
\begin{aligned}
\int_{0}^{T} \int_{\Omega}\left|\varepsilon \nabla_{x} \mathbf{v}^{\varepsilon}\right|^{r} \mathrm{~d} x \mathrm{~d} t & =\int_{0}^{T} \int_{\Omega} \varepsilon^{\frac{r}{2}+\frac{r}{2}}\left|\nabla_{x} \mathbf{v}^{\varepsilon}\right|^{r} \mathrm{~d} x \mathrm{~d} t \\
& \leq \varepsilon^{\frac{r}{2}}\left(\int_{0}^{T} \int_{\Omega} \varepsilon\left|\nabla_{x} \mathbf{v}^{\varepsilon}\right|^{2} \mathrm{~d} x \mathrm{~d} t\right)^{\frac{2}{r}}\left|\Omega_{T}\right|^{\frac{2}{2-r}} \\
\int_{0}^{T} \int_{\Omega}\left(\varepsilon\left|\mathbb{D}_{x} \mathbf{v}^{\varepsilon}\right|^{\frac{6}{5}}\right)^{r} \mathrm{~d} x \mathrm{~d} t & =\int_{0}^{T} \int_{\Omega} \varepsilon^{\frac{5 r}{11}+\frac{6 r}{11}}\left|\mathbb{D}_{x} \mathbf{v}^{\varepsilon}\right|^{\frac{6 r}{5}} \mathrm{~d} x \mathrm{~d} t \\
& \leq \varepsilon^{\frac{5 r}{11}}\left(\int_{0}^{T} \int_{\Omega} \varepsilon\left|\mathbb{D}_{x} \mathbf{v}^{\varepsilon}\right|^{\frac{11}{5}} \mathrm{~d} x \mathrm{~d} t\right)^{\frac{6 r}{11}}\left|\Omega_{T}\right|^{\frac{11}{11-6 r}}
\end{aligned}
$$

thus using also (2.49) we get that for any $r<5 q / 6$

$$
\begin{aligned}
\mathbb{H}_{2}^{\varepsilon}:= & \varepsilon \nabla_{x} \mathbf{v}^{\varepsilon}+\varepsilon\left|\mathbb{D}_{x} \mathbf{v}^{\varepsilon}\right|^{\frac{1}{5}} \mathbb{D}_{x} \mathbf{v}^{\varepsilon}+\left(\mathbf{v} \otimes \mathbf{v}-\mathbf{v}^{\varepsilon} \otimes \mathbf{v}^{\varepsilon}\right) \\
& \rightarrow 0 \text { strongly in } L^{r}\left(\Omega_{T} ; R^{3 \times 3}\right) \text { as } \varepsilon \rightarrow 0 .
\end{aligned}
$$

Therefore, (2.81) can be rewritten again as:

$$
\int_{0}^{T} \int_{\Omega} \mathbf{u}^{\varepsilon} \cdot \partial_{t} \boldsymbol{\varphi} \mathrm{d} x \mathrm{~d} t=\int_{0}^{T} \int_{\Omega}\left(\mathbb{H}_{1}^{\varepsilon}+\mathbb{H}_{2}^{\varepsilon}\right): \mathbb{D}_{x} \boldsymbol{\varphi} \mathrm{d} x \mathrm{~d} t
$$

for any $\varphi \in C^{\infty}\left(0, T ; C_{0, \text { div }}^{\infty}\left(\Omega ; R^{3}\right)\right)$. At this point let us set $\varepsilon:=\varepsilon_{m}=1 / m$. Then all the assumptions of Theorem 4.1 and Corollary 4.2 in Sect. 4 are fulfilled. Particularly, note that we can consider any small $r>1$. Now, take any cylinder $Q \subset(0, T) \times \Omega$, for suitable $\xi \in C_{0}^{\infty}\left(\frac{1}{6} Q ; R^{3}\right),{ }^{1}$ for any $\theta \in(0,1)$, set $\mathbb{S}:=\left|\mathbb{D}_{x} \mathbf{v}\right|^{q-2} \mathbb{D}_{x} \mathbf{v}$, then consider the following quantity

\footnotetext{
${ }^{1}$ For any $\alpha>0$ we denote $\alpha Q$ the cylinder $Q$ scaled by $\alpha$ with respect to its center.
} 


$$
\begin{aligned}
\int_{Q} & \left(\left(\mathbb{S}^{m}-\mathbb{S}\right):\left(\mathbb{D}_{x} \mathbf{v}^{m}-\mathbb{D}_{x} \mathbf{v}\right)\right)^{\theta} \xi \mathrm{d} x \mathrm{~d} t \\
= & \int_{Q}\left(\left(\mathbb{S}^{m}-\mathbb{S}\right):\left(\mathbb{D}_{x} \mathbf{v}^{m}-\mathbb{D}_{x} \mathbf{v}\right)\right)^{\theta} \xi \chi_{O_{m, k}} \mathrm{~d} x \mathrm{~d} t \\
& \quad+\int_{Q}\left(\left(\mathbb{S}^{m}-\mathbb{S}\right):\left(\mathbb{D}_{x} \mathbf{v}^{m}-\mathbb{D}_{x} \mathbf{v}\right)\right)^{\theta} \xi \chi_{O_{m, k}^{C}} \mathrm{~d} x \mathrm{~d} t
\end{aligned}
$$

Let us treat the two terms separately. For the first term using the Hölder inequality, the established uniform estimates in $\varepsilon_{m}$, the statements (a) and (h) of Theorem 4.1 we obtain

$$
\begin{aligned}
& \limsup _{m \rightarrow+\infty} \int_{Q}\left(\left(\mathbb{S}^{m}-\mathbb{S}\right):\left(\mathbb{D}_{x} \mathbf{v}^{m}-\mathbb{D}_{x} \mathbf{v}\right)\right)^{\theta} \xi \chi_{O_{m, k}} \mathrm{~d} x \mathrm{~d} t \\
& \quad \leq C \limsup _{m \rightarrow+\infty}\left(\int_{Q}\left(\mathbb{S}^{m}-\mathbb{S}\right):\left(\mathbb{D}_{x} \mathbf{v}^{m}-\mathbb{D}_{x} \mathbf{v}\right) \mathrm{d} x \mathrm{~d} t\right)^{\theta}\left|O_{m, k}\right|^{1-\theta} \\
& \quad \leq C 2^{k(\theta-1)} .
\end{aligned}
$$

On the other hand, in the second term Hölder's inequality gives

$$
\begin{aligned}
& \int_{Q}\left(\left(\mathbb{S}^{m}-\mathbb{S}\right):\left(\mathbb{D}_{x} \mathbf{v}^{m}-\mathbb{D}_{x} \mathbf{v}\right)\right)^{\theta} \xi \chi_{O_{m, k}^{C}} \mathrm{~d} x \mathrm{~d} t \\
& =\int_{O_{m, k}^{C}}\left(\left(\mathbb{S}^{m}-\mathbb{S}\right):\left(\mathbb{D}_{x} \mathbf{v}^{m}-\mathbb{D}_{x} \mathbf{v}\right) \xi\right)^{\theta} \xi^{1-\theta} \mathrm{d} x \mathrm{~d} t \\
& \quad \leq C\left(\int_{Q}\left(\mathbb{S}^{m}-\mathbb{S}\right):\left(\mathbb{D}_{x} \mathbf{v}^{m}-\mathbb{D}_{x} \mathbf{v}\right) \xi \chi_{O_{m, k}^{C}} \mathrm{~d} x \mathrm{~d} t\right)^{\theta}
\end{aligned}
$$

Setting $\mathbb{K}:=\overline{\mathbb{S}}-\mathbb{S}$ it results true the identity

$$
\mathbb{S}^{m}-\mathbb{S}=\mathbb{H}_{1}^{m}+\mathbb{K}
$$

and employing Corollary 4.2 it follows that

$$
\limsup _{m \rightarrow+\infty} \int_{Q}\left(\left(\mathbb{S}^{m}-\mathbb{S}\right):\left(\mathbb{D}_{x} \mathbf{v}^{m}-\mathbb{D}_{x} \mathbf{v}\right)\right)^{\theta} \xi \chi_{O_{m, k}^{C}} \mathrm{~d} x \mathrm{~d} t \leq C 2^{-\frac{k \theta}{q}} .
$$

Gathering (2.88) and (2.89) it results that

$$
\limsup _{m \rightarrow+\infty} \int_{Q}\left(\left(\mathbb{S}^{m}-\mathbb{S}\right):\left(\mathbb{D}_{x} \mathbf{v}^{m}-\mathbb{D}_{x} \mathbf{v}\right)\right)^{\theta} \xi \mathrm{d} x \mathrm{~d} t \leq C 2^{k(\theta-1)}+C 2^{-\frac{k \theta}{q}},
$$

and since $k$ can be chosen arbitrarily large finally we have that

$$
\begin{aligned}
& \limsup _{m \rightarrow+\infty} \int_{\frac{1}{8} Q}\left(\left(\mathbb{S}^{m}-\mathbb{S}\right):\left(\mathbb{D}_{x} \mathbf{v}^{m}-\mathbb{D}_{x} \mathbf{v}\right)\right)^{\theta} \mathrm{d} x \mathrm{~d} t \\
& \quad \leq \limsup _{m \rightarrow+\infty} \int_{Q}\left(\left(\mathbb{S}^{m}-\mathbb{S}\right):\left(\mathbb{D}_{x} \mathbf{v}^{m}-\mathbb{D}_{x} \mathbf{v}\right)\right)^{\theta} \xi \mathrm{d} x \mathrm{~d} t=0 .
\end{aligned}
$$

By the monotonicity of the integrand on the left-hand side it follows that

$$
\lim _{m \rightarrow+\infty}\left(\mathbb{S}^{m}-\mathbb{S}\right):\left(\mathbb{D}_{x} \mathbf{v}^{m}-\mathbb{D}_{x} \mathbf{v}\right)=0 \text { a.e. in } \frac{1}{8} Q,
$$

thus by virtue of the results of Dal Maso and Murat [11] we conclude that

$$
\lim _{m \rightarrow+\infty} \mathbb{D}_{x} \mathbf{v}^{m}=\mathbb{D}_{x} \mathbf{v} \text { a.e. in } \frac{1}{8} Q,
$$

and then the continuity of the stress tensor implies that

$$
\lim _{m \rightarrow+\infty} \mathbb{S}^{m}=\mathbb{S}=\left|\mathbb{D}_{x} \mathbf{v}\right|^{q-2} \mathbb{D}_{x} \mathbf{v} \text { a.e. in } \frac{1}{8} Q .
$$


Since $Q$ was arbitrary we can conclude that

$$
\lim _{m \rightarrow+\infty} \mathbb{S}^{m}=\mathbb{S}=\left|\mathbb{D}_{x} \mathbf{v}\right|^{q-2} \mathbb{D}_{x} \mathbf{v} \text { a.e. in } \Omega_{T} .
$$

Being $\mathbb{S}^{m}$ uniformly integrable, the Vitali convergence theorem yields $\mathbb{S}^{m} \rightarrow \mathbb{S}$ weakly in $L^{q^{\prime}}\left(\Omega_{T} ; R^{3 \times 3}\right)$ and finally

$$
\overline{\mathbb{S}}=\mathbb{S}=\left|\mathbb{D}_{x} \mathbf{v}\right|^{q-2} \mathbb{D}_{x} \mathbf{v} \text { a.e. in } \Omega_{T}
$$

The proof is complete.

\section{Proof of Corollary 1.5}

By virtue of the proof of Theorem 1.3, we can claim that for any $\varepsilon>0$ there exists $\mathbf{v}^{\varepsilon}$ and $\mathbb{S}^{\varepsilon}$ such that

$$
\begin{aligned}
\operatorname{int}_{0}^{\tau}\left\langle\partial_{t} \mathbf{v}^{\varepsilon}, \boldsymbol{\varphi}\right\rangle \mathrm{d} t-\int_{0}^{\tau} \int_{\Omega} \mathbf{v}^{\varepsilon} \otimes \mathbf{v}^{\varepsilon}: \nabla_{x} \boldsymbol{\varphi} \mathrm{d} x \mathrm{~d} t+\int_{0}^{\tau} \int_{\Omega} \mathbb{S}^{\varepsilon}: \mathbb{D}_{x} \boldsymbol{\varphi} \mathrm{d} x \mathrm{~d} t \\
\quad+\varepsilon \int_{0}^{\tau} \int_{\Omega} \nabla_{x} \mathbf{v}^{\varepsilon}: \nabla_{x} \boldsymbol{\varphi} \mathrm{d} x \mathrm{~d} t+\varepsilon \int_{0}^{\tau} \int_{\Omega}\left|\mathbb{D}_{x} \mathbf{v}^{\varepsilon}\right|^{\frac{1}{5}} \mathbb{D}_{x} \mathbf{v}^{\varepsilon}: \mathbb{D}_{x} \boldsymbol{\varphi} \mathrm{d} x \mathrm{~d} t \\
=\int_{0}^{\tau} \int_{\Omega} \mathbf{b} \cdot \boldsymbol{\varphi} \mathrm{d} x \mathrm{~d} t \text { for any } \boldsymbol{\varphi} \in L^{\frac{11}{5}}\left(0, T ; W_{0, \operatorname{div}}^{1, \frac{11}{5}}\left(\Omega ; R^{3}\right)\right),
\end{aligned}
$$

for any $0<\tau \leq T$, with

$$
\mathbb{S}^{\varepsilon}=\left|\mathbb{D}_{x} \mathbf{v}^{\varepsilon}\right|^{q-2} \mathbb{D}_{x} \mathbf{v}^{\varepsilon} \text { a.e. in } \Omega_{T},
$$

and

$$
\mathbf{v}^{\varepsilon}(T)=\mathbf{v}^{\varepsilon}(0) \text { in } L^{2}\left(\Omega ; R^{3}\right) .
$$

Let us consider $\varphi=\mathbf{v}^{\varepsilon}$ in (3.1) it follows that

$$
\begin{aligned}
& \frac{1}{2} \frac{\mathrm{d}}{\mathrm{d} t}\left\|\mathbf{v}^{\varepsilon}(t)\right\|_{L^{2}\left(\Omega ; R^{3}\right)}^{2}+\left\|\mathbb{D}_{x} \mathbf{v}^{\varepsilon}(t)\right\|_{L^{q}\left(\Omega ; R^{3 \times 3}\right)}^{q} \\
& \quad \leq\|\mathbf{b}(t)\|_{L^{2}\left(\Omega ; R^{3}\right)}\left\|\mathbf{v}^{\varepsilon}(t)\right\|_{L^{2}\left(\Omega ; R^{3}\right)} \\
& \quad \leq C\|\mathbf{b}(t)\|_{L^{2}\left(\Omega ; R^{3}\right)}^{q^{\prime}}+\frac{1}{2}\left\|\mathbb{D}_{x} \mathbf{v}^{\varepsilon}(t)\right\|_{L^{q}\left(\Omega ; R^{3 \times 3}\right)}^{q}
\end{aligned}
$$

for a.a. $t \in(0, T)$, then

$$
\frac{1}{2} \frac{\mathrm{d}}{\mathrm{d} t}\left\|\mathbf{v}^{\varepsilon}(t)\right\|_{L^{2}\left(\Omega ; R^{3}\right)}^{2}+\alpha\left\|\mathbf{v}^{\varepsilon}(t)\right\|_{L^{2}\left(\Omega ; R^{3}\right)}^{q} \leq C\|\mathbf{b}(t)\|_{L^{2}\left(\Omega ; R^{3}\right)}^{q^{\prime}}
$$

for a.a. $t \in(0, T)$, where $\alpha>0$ is the constant due to the Sobolev embedding $W_{0}^{1, q} \hookrightarrow L^{2}$, while $C>0$ is a constant that may vary from line to line but it is independent of $\varepsilon$ and of $t$. Since $\|\mathbf{b}(t)\|_{L^{2}\left(\Omega ; R^{3}\right)}=0$ in $[\bar{t}, T],(3.3)$ implies that

$$
\frac{1}{2} \frac{\mathrm{d}}{\mathrm{d} t}\left\|\mathbf{v}^{\varepsilon}(t)\right\|_{L^{2}\left(\Omega ; R^{3}\right)}^{2}+\alpha\left\|\mathbf{v}^{\varepsilon}(t)\right\|_{L^{2}\left(\Omega ; R^{3}\right)}^{q} \leq 0 \text { for a.a. } t \in(\bar{t}, T) .
$$

Restricting $q \in(6 / 5 ; 2)$, integrating between $\bar{t}$ and $t$, and employing (2.59) we get

$$
\begin{aligned}
\left\|\mathbf{v}^{\varepsilon}(t)\right\|_{L^{2}\left(\Omega ; R^{3}\right)}^{2-q} & \leq\left\|\mathbf{v}^{\varepsilon}(0)\right\|_{L^{2}\left(\Omega ; R^{3}\right)}^{2-q}-(2-q) \alpha(t-\bar{t}) \\
& \leq \bar{K}^{2-q}-(2-q) \alpha(t-\bar{t}),
\end{aligned}
$$

therefore

$$
\left\|\mathbf{v}^{\varepsilon}(t)\right\|_{L^{2}\left(\Omega ; R^{3}\right)}=0 \text { for a.a. } t: \bar{t}+\frac{\bar{K}^{2-q}}{\alpha(2-q)} \leq t \leq T \text {. }
$$


Now let us follow the limit as $\varepsilon \rightarrow 0$ as showed in the previous section. In particular it holds

$$
\mathbf{v}^{\varepsilon}(t) \rightarrow \mathbf{v}(t) \text { weakly in } L^{2}\left(\Omega ; R^{3}\right) \text { for a.a. } t \in\left(\bar{t}_{v}, T\right)
$$

where

$$
\bar{t}_{v}:=\bar{t}+\frac{\bar{K}^{2-q}}{\alpha(2-q)},
$$

then the weak-lower semicontinuity of the $L^{2}$-norm gives that

$$
\|\mathbf{v}(t)\|_{L^{2}\left(\Omega ; R^{3}\right)} \leq \liminf _{\varepsilon \rightarrow 0}\left\|\mathbf{v}^{\varepsilon}(t)\right\|_{L^{2}\left(\Omega ; R^{3}\right)}=0 \text { for a.a. } t \in\left(\bar{t}_{v}, T\right)
$$

and this finishes the proof.

\section{Auxilliary Tools}

We state the divergence-free Lipschitz truncations of Bochner-Sobolev functions taken from [7].

Theorem 4.1. Let $Q=I \times B \subset R \times R^{3}$ be a space-time cylinder and let $1<q<+\infty$ with $q, q^{\prime}:=$ $q /(q-1)>r>1$. Let $\left\{\mathbf{u}^{m}\right\}$ and $\left\{\mathbb{H}^{m}\right\}$ with $\mathbb{H}^{m}:=\mathbb{H}_{1}^{m}+\mathbb{H}_{2}^{m}$ be sequences fulfilling

$$
\begin{aligned}
& \operatorname{div}_{x} \mathbf{u}^{m}=0 \text { a.e. in } Q, \\
& \partial_{t} \mathbf{u}^{m}=-\operatorname{div}_{x} \mathbb{H}^{m} \text { in sense of distributions in } C^{\infty}\left(I ; C_{0, \operatorname{div}}^{\infty}\left(B ; R^{3}\right)\right),
\end{aligned}
$$

assume that $\mathbf{u}^{m}$ is bounded in $L^{\infty}\left(I ; L^{r}\left(B ; R^{3}\right)\right)$ uniformly and that

$$
\begin{aligned}
\mathbf{u}^{m} \rightarrow 0 & \text { weakly in } L^{q}\left(I ; W_{0}^{1, q}\left(B ; R^{3}\right)\right), \\
\mathbf{u}^{m} \rightarrow 0 & \text { strongly in } \left.L^{r}\left(Q ; R^{3}\right)\right), \\
\mathbb{H}_{1}^{m} \rightarrow 0 & \text { weakly in } L^{q^{\prime}}\left(Q ; R^{3 \times 3}\right), \\
\mathbb{H}_{2}^{m} \rightarrow 0 & \text { strongly in } L^{r}\left(Q ; R^{3 \times 3}\right)
\end{aligned}
$$

as $m \rightarrow+\infty$. Then, there is a double sequence $\left\{\lambda_{m, k}\right\}_{m, k=1}^{\infty} \subset(0, \infty)$ such that

(a) $2^{2^{k}} \leq \lambda_{m, k} \leq 2^{2^{k+1}}$,

there exist a double sequence of functions $\left\{\mathbf{u}^{m, k}\right\}_{m, k=1}^{\infty} \subset L^{1}\left(Q ; R^{3}\right)$, a double sequence $\left\{O_{m, k}\right\}_{m, k=1}^{\infty}$ of measurable subsets of $Q$, a constant $C>0$ and $k_{0} \in N$ such that for any $k \geq k_{0}$ the following properties are satisfied:

(b) $\mathbf{u}^{m, k} \in L^{s}\left(\frac{1}{4} I ; W_{0, \operatorname{div}}^{1, s}\left(\frac{1}{6} B ; R^{3}\right)\right)$ for any $s \in(1, \infty)$,

(c) $\operatorname{supp} \mathbf{u}^{m, k} \subset \frac{1}{6} Q$,

(d) $\mathbf{u}^{m, k}=\mathbf{u}^{m}$ a.e. in $\frac{1}{8} Q \backslash O_{m, k}$,

(e) $\left\|\nabla_{x} \mathbf{u}^{m, k}\right\|_{L^{\infty}\left(\frac{1}{4} Q ; R^{3 \times 3}\right)} \leq C \lambda_{m, k}$,

(f) $\mathbf{u}^{m, k} \rightarrow 0$ strongly in $L^{\infty}\left(\frac{1}{4} Q ; R^{3}\right)$ as $m \rightarrow \infty$,

(g) $\nabla_{x} \mathbf{u}^{m, k} \rightarrow 0$ weakly-* in $L^{\infty}\left(\frac{1}{4} Q ; R^{3}\right)$ as $m \rightarrow \infty$,

(h) $\lim \sup _{m \rightarrow+\infty}\left(\lambda_{m, k}\right)^{q}\left|O_{m, k}\right| \leq C 2^{-k}$,

(i) $\lim \sup _{m \rightarrow+\infty}\left|\int_{\frac{1}{8} Q \backslash O^{m, k}} \mathbb{H}^{m}: \nabla_{x} \mathbf{u}^{m, k} \mathrm{~d} x \mathrm{~d} t\right| \leq C\left(\lambda_{m, k}\right)^{q}\left|O_{m, k}\right|$.

Corollary 4.2. Let all the assumptions of Theorem 4.1 be fulfilled and assume that $\mathbf{u}^{m}$ is bounded in $L^{\infty}\left(I ; L^{r}\left(B ; R^{3}\right)\right)$ uniformly in $m$. Then there exist $\xi \in C_{0}^{\infty}\left(\frac{1}{6} Q ; R^{3}\right)$ such that $\chi_{\frac{1}{8} Q} \leq \xi \leq \chi_{\frac{1}{6} Q}$ and a constant $C$ depending only on $\xi$ such that for every $\mathbb{K} \in L^{q^{\prime}}\left(\frac{1}{6} Q ; R^{3 \times 3}\right)$ there holds:

$$
\limsup _{m \rightarrow+\infty}\left|\int\left(\mathbb{H}_{1}^{m}+\mathbb{K}\right): \nabla_{x} \mathbf{u}^{m} \xi \chi_{O_{m, k}^{c}} \mathrm{~d} x \mathrm{~d} t\right| \leq C 2^{-\frac{k}{q}},
$$


and

$$
\begin{aligned}
& \mathbf{u}^{m, k} \rightarrow 0 \text { strongly in } L^{s}\left(\frac{1}{4} Q ; R^{3}\right) \text { as } m \rightarrow \infty \text { and } k \text { fixed, } \forall s \in(1, \infty), \\
& \nabla_{x} \mathbf{u}^{m, k} \rightarrow 0 \text { weakly in } L^{s}\left(\frac{1}{4} Q ; R^{3}\right) \text { as } m \rightarrow \infty \text { and } k \text { fixed, } \forall s \in(1, \infty) .
\end{aligned}
$$

Acknowledgements. The research of A.A. is supported by Einstein Foundation, Berlin. She is also member of the Italian National Group for the Mathematical Physics (GNFM) of INdAM.

Funding Open Access funding enabled and organized by Projekt DEAL.

\section{Declarations}

Conflict of interest The author states that there is no conflict of interest.

Open Access. This article is licensed under a Creative Commons Attribution 4.0 International License, which permits use, sharing, adaptation, distribution and reproduction in any medium or format, as long as you give appropriate credit to the original author(s) and the source, provide a link to the Creative Commons licence, and indicate if changes were made. The images or other third party material in this article are included in the article's Creative Commons licence, unless indicated otherwise in a credit line to the material. If material is not included in the article's Creative Commons licence and your intended use is not permitted by statutory regulation or exceeds the permitted use, you will need to obtain permission directly from the copyright holder. To view a copy of this licence, visit http://creativecommons.org/licenses/by/4.0/.

Publisher's Note Springer Nature remains neutral with regard to jurisdictional claims in published maps and institutional affiliations.

\section{References}

[1] Abbatiello, A., Feireisl, E.: On a class of generalized solutions to equations describing incompressible viscous fluids. Ann. Mat. Pura Appl. (4) 199(3), 1183-1195 (2020)

[2] Abbatiello, A., Crispo, F., Maremonti, P.: Electrorheological fluids: ill posedness of uniqueness backward in time. Nonlinear Anal. 170, 47-69 (2018)

[3] Abbatiello, A., Maremonti, P.: Existence of regular time-periodic solutions to shear-thinning fluids. J. Math. Fluid Mech. 21(2), 14 (2019)

[4] Axmann, Š, Pokorný, M.: Time-periodic solutions to the full Navier-Stokes-Fourier system with radiation on the boundary. J. Math. Anal. Appl. 428(1), 414-444 (2015)

[5] Barhoun, A., Lemlih, A.B.: A reproductive property for a class of non-Newtonian fluids. Appl. Anal. 81(1), 13-38 (2002)

[6] Blechta, J., Málek, J., Rajagopal, K.R.: On the classification of incompressible fluids and a mathematical analysis of the equations that govern their motion. SIAM J. Math. Anal. 52(2), 1232-1289 (2020)

[7] Breit, D., Diening, L., Schwarzacher, S.: Solenoidal Lipschitz truncation for parabolic PDEs. Math. Models Methods Appl. Sci. 23(14), 2671-2700 (2013)

[8] Burczak, J., Modena, S., Székelyhidi, L.: Non-uniqueness of power-law flows. Arxiv Preprint Series arXiv:2007.08011 (2020)

[9] Crispo, F.: A note on the existence and uniqueness of time-periodic electro-rheological flows. Acta Appl. Math. 132, $237-250(2014)$

[10] Crispo, F., Grisanti, C., Maremonti, P.: Singular p-Laplacian parabolic system in exterior domains: higher regularity of solutions and related properties of extinction and asymptotic behavior in time. Ann. Sc. Norm. Super. Pisa Cl. Sci. (5) 19(3), 913-949 (2019)

[11] Dal Maso, G., Murat, F.: Almost everywhere convergence of gradients of solutions to nonlinear elliptic systems. Nonlinear Anal. 31(3-4), 405-412 (1998)

[12] DiBenedetto, E.: Degenerate parabolic equations. Universitext. Springer, New York (1993)

[13] Diening, L., Růžička, M., Wolf, J.: Existence of weak solutions for unsteady motions of generalized Newtonian fluids. Ann. Sc. Norm. Super. Pisa Cl. Sci. (5) 9(1), 1-46 (2010)

[14] Feireisl, E., Matušu-Nečasová, Š, Petzeltová, H., Straškraba, I.: On the motion of a viscous compressible flow driven by a time-periodic external flow. Arch. Ration. Mech. Anal. 149, 69-96 (1999)

[15] Feireisl, E., Mucha, P., Novotný, A., Pokorný, M.: Time periodic solutions to the full Navier-Stokes-Fourier system. Arch. Ration. Mech. Anal. 204, 745-786 (2012)

[16] Galdi, G.P.: Existence and uniqueness of time-periodic solutions to the Navier-Stokes equations in the whole plane. Discrete Contin. Dyn. Syst. Ser. S 6(5), 1237-1257 (2013) 
[17] Galdi, G.P., Grisanti, C.R.: Womersley flow of generalized Newtonian liquid. Proc. R. Soc. Edinb. Sect. A 146(4), 671-692 (2016)

[18] Galdi, G.P., Kyed, M.: Time-periodic solutions to the Navier-Stokes equations. In: Giga, Y., Novotný, A. (eds.) Handbook of Mathematical Analysis in Mechanics of Viscous Fluids, pp. 509-578. Springer, Cham (2018)

[19] Lions, J.L.: Sur certaines équations paraboliques non linéaires. Bull. Soc. Math. France 93, 155-175 (1965)

[20] Málek, J., Nečas, J., Rokyta, M., Růžička, M.: Weak and Measure-Valued Solutions to Evolutionary PDEs, Applied Mathematics and Mathematical Computation, vol. 13. Chapman \& Hall, London (1996)

[21] Málek, J., Rajagopal, K.R.: Mathematical issues concerning the Navier-Stokes equations and some of its generalizations. In: Dafermos, C.M., Feireisl, E. (eds.) Handbook of Differential Equations: Evolutionary Equations, vol. II, pp. 371-459. Elsevier, Amsterdam (2005)

[22] Maremonti, P.: Existence and stability of time-periodic solutions to the Navier-Stokes equations in the whole space. Nonlinearity 4(2), 503-529 (1991)

[23] Maremonti, P., Padula, M.: Existence, uniqueness and attainability of periodic solutions of the Navier-Stokes equations in exterior domains, Zap. Nauchn. Sem. S.-Peterburg. Otdel. Mat. Inst. Steklov. (POMI) 233 (1996), no. Kraev. Zadachi Mat. Fiz. i Smezh. Vopr. Teor. Funkts. 27, 142-182, 257

[24] Prouse, G.: Soluzioni periodiche dell'equazione di Navier-Stokes. Atti Accad. Naz. Lincei Rend. Cl. Sci. Fis. Mat. Nat. (8) 35, 443-447 (1963)

Anna Abbatiello

Institute of Mathematics

Technische Universität Berlin

Straße des 17. Juni 136

10623 Berlin

Germany

e-mail: anna.abbatiello@tu-berlin.de

(accepted: January 30, 2021; published online: May 20, 2021) 